\title{
Starvation causes changes in the intestinal transcriptome and microbiome that are reversed upon refeeding
}

Jayanth Jawahar

Duke University

Alexander McCumber

Duke University

Colin Lickwar

Duke University

Caroline Amoroso

Duke University

Sol Gomez de la Torre Canny

Duke University

\section{Sandi Wong}

Duke University

\section{Brendan Bohannan}

University of Oregon

Karen Guillemin

University of Oregon

John Rawls ( $\square$ john.rawls@duke.edu )

Duke University

\section{Research Article}

Keywords: starvation, microbiome, refeeding, homeostasis

Posted Date: December 17th, 2020

DOl: https://doi.org/10.21203/rs.3.rs-120052/v1

License: (c) (1) This work is licensed under a Creative Commons Attribution 4.0 International License. Read Full License

Version of Record: A version of this preprint was published at BMC Genomics on March 22nd, 2022. See the published version at https://doi.org/10.1186/s12864-022-08447-2. 

1 Starvation causes changes in the intestinal transcriptome and microbiome that are reversed upon refeeding

Jayanth Jawahar ${ }^{* 1}$, Alexander W. McCumber², Colin R. Lickwar ${ }^{1}$, Caroline R. Amoroso ${ }^{3}$, Sol Gomez de la Torre Canny ${ }^{1}$, Sandi Wong ${ }^{1}$, Brendan J. M. Bohannan ${ }^{4}$, Karen Guillemin $^{5}$, and John F. Rawls ${ }^{1^{* *}}$

1 Department of Molecular Genetics and Microbiology, Duke Microbiome Center, Duke University School of Medicine, Durham, NC 27710

${ }^{2}$ Civil and Environmental Engineering Department, Duke University, Durham, NC 27708

${ }^{3}$ Department of Evolutionary Anthropology, Duke University, Durham, NC 27708

${ }^{4}$ Institute of Ecology and Evolution, University of Oregon, Eugene, OR 97403

${ }^{5}$ Institute of Molecular Biology, University of Oregon, Eugene, OR 97403

* authors contributed equally

${ }^{* *}$ Correspondence to john.rawls@duke.edu

\section{Abstract:}

\section{Background:}

The ability of animals and their microbiomes to adapt to starvation and then restore homeostasis after refeeding is fundamental to their continued survival and symbiosis. The intestine is the primary site of nutrient absorption and microbiome interaction, however our understanding of intestinal adaptations in host transcriptional programs and microbiome composition remains limited. Additionally, few studies on starvation have investigated intestinal responses to refeeding. The zebrafish presents unique opportunities to study the effects of long-term starvation and refeeding. We used RNA sequencing and 16S rRNA gene sequencing to uncover changes in the intestinal transcriptome and microbiome of zebrafish subjected to long-term starvation and refeeding compared to continuously fed controls.

\section{Results:}

Starvation over 21 days led to increased diversity and altered composition in the intestinal microbiome compared to fed controls, including relative increases in Vibrio and reductions in Plesiomonas bacteria. Starvation also led to significant alterations in host gene expression in the intestine, with distinct pathways affected at early and late stages of starvation. This included increases in the expression of ribosome biogenesis genes early in starvation, followed by decreased expression of genes involved in antiviral immunity and lipid transport at later stages. These effects of starvation on the host transcriptome and microbiome were almost completely restored within 3 days after refeeding. 
Comparison with published datasets identified host genes responsive to starvation as well as high-fat feeding or microbiome colonization, and predicted host transcription factors that may be involved in starvation response.

\section{Conclusions:}

Long-term starvation induces progressive changes in microbiome composition and host gene expression in the zebrafish intestine, and these changes are rapidly reversed after refeeding. Our identification of bacterial taxa, host genes and host pathways involved in this response provides a framework for future investigation of the physiological and ecological mechanisms underlying intestinal adaptations to food restriction.

\section{Introduction}

Starvation is a state of severe caloric restriction regularly experienced by many animal species and a significant portion of the human population. In humans, starvation can be the result of environmental or socioeconomic conditions including war, famine, and poverty ${ }^{1}$. It can also occur alongside pathologies such as anorexia nervosa and cancer ${ }^{2}$. In animals, periods of absolute or relative starvation can result from seasonal changes such as drought and severe cold, or from behaviors such as nesting, lactation, migration, and hibernation ${ }^{3}$. This wide range of circumstances leading to starvation across the animal kingdom evokes a range of progressive physiologic adaptations to starvation across different species. Indeed, previous studies have reported similarity and divergence in starvation physiology across animal taxa such as humans, rodents, polar bears, penguins, reptiles, amphibians, fish, and insects ${ }^{4}$. However, previous studies have largely focused on tissue histopathologies associated with starvation, whereas effects on the underlying physiological processes (mediated both by the host and its microbiome) remain incompletely understood.

Across many animal species, starvation leads to a progressive decrease in metabolic rate ${ }^{5}$. Increased blood glycerol, which serves as a gluconeogenic precursor, is also common in starved animals, as are fluctuations in free fatty acids ${ }^{3,4}$. The overall depletion in energy stores leads to weight loss, which is generally greater in endotherms when compared to ectotherms ${ }^{6}$. Starvation is also associated with a gradual reduction in mass in important organs such as the liver, skeletal muscle, and intestine ${ }^{4,7}$. These effects necessitate a recovery from starvation to restore optimal function to these organs.

Inherently linked to starvation, the return to homeostasis in different species following starvation is facilitated by a refeeding response that gradually reverses starvation-induced adaptations and restores energy balance. Physiological responses to starvation and subsequent refeeding are dynamic and complex, involving coordination 
of these responses is often the preservation of lean body mass while favoring the depletion of energy stores such as glycogen and fat ${ }^{8,9}$. However, despite these effects, starvation often results in lasting defects on bone density, pancreatic function, and mental development long after refeeding ${ }^{10-12}$. Thus, improved understanding of these dynamic physiological processes could lead to new approaches to reduce morbidities and mortalities associated with starvation in humans and other animals ${ }^{13}$.

Previous studies on the effects of refeeding after starvation have largely focused on tissues such as liver, skeletal muscle, brain, and pancreatic islets ${ }^{14-18}$. We have a relatively poor understanding of the transcriptional starvation and refeeding responses in the intestine. The intestine is the major site of dietary nutrient sensing and absorption, and harbors complex communities of microorganisms (aka the gut microbiome). Previous studies in humans and rodent models have shown that intestinal microbiome composition changes in response to starvation and diet composition with distinct contributions to the nutritional physiology of their hosts ${ }^{19-26}$. These findings informed more recent studies that have investigated microbiome-targeted therapeutics for alleviating starvation and its associated developmental defects ${ }^{27-29}$. However, gut microbial responses to starvation have been largely limited to mammals, and our understanding of intestinal physiological responses to starvation and feeding in any animal remains quite limited.

Animal models provide opportunities to study the processes that underlie starvation and refeeding responses in vertebrates, resulting in a general understanding that may be translated to humans ${ }^{30}$. Poikilothermic vertebrates such as cyprinid fishes are particularly interesting due to their capacity to endure prolonged starvation periods. In response to prolonged starvation, cyprinids such as carp exhibit a reduction in intestinal thickness and weight, altered enterocyte morphology, and a decrease in body weight and liver size, similar to the starvation response in mice ${ }^{17,31-35}$. Zebrafish (Danio rerio) survive up to 4 weeks of starvation, and a suite of genomic and genetic resources facilitate the investigation of their physiology ${ }^{36}$. Using in vivo imaging to monitor white adipose tissues as a measure of energy storage, we previously showed that prolonged starvation in adult zebrafish leads to progressive mobilization of fat stored in white adipose tissues, which is replenished in response to refeeding ${ }^{37-39}$. Because adipose tissues develop progressively during juvenile and adult stages, the duration of starvation required to completely mobilize adipose lipid increases with animal age (e.g., from 1 week in juveniles up to 3 weeks in adults) ${ }^{37-39}$. However, the impact of prolonged starvation and refeeding on the zebrafish intestine has not been explored.

The zebrafish intestine displays extensive cellular and physiological homology to that of mammals, and harbors a microbiome that varies in composition as a function of age and diet composition ${ }^{40-45}$. The presence and composition of the intestinal microbiome in zebrafish impacts the host by regulating dietary nutrient absorption, epithelial renewal, and inflammation ${ }^{41,43,46-50}$. By comparing patterns of gene expression and accessible chromatin in intestinal epithelial cells from zebrafish, mouse, and human, 
121 we recently discovered a conserved transcriptional regulatory network conserved across

122420 million years of vertebrate evolution ${ }^{51}$. Building upon this recent work, here we define 123 the impact of prolonged starvation and refeeding on gene expression in the zebrafish 124 intestine, and use these results to predict the physiological processes and transcription 125 regulatory pathways that underlie the response to starvation and refeeding. We also show how the taxonomic composition of the zebrafish intestinal microbiome is altered during the same prolonged starvation and refeeding regimen.

\section{Results}

\section{Starvation is accompanied by significant changes in gut microbiome composition that are reversed during refeeding}

To determine the influence of starvation on zebrafish microbiome composition and intestinal gene expression, zebrafish were reared under conventional conditions using a standard diet to early adulthood (60 days post fertilization or dpf). Animals were then moved into clean tanks and randomly assigned into one of two treatment groups: one group was starved for 21 days followed by 21 days refeeding, and a reference control group was consistently fed across the same 42-day time course (Fig. 1A). This 21 day starvation regimen was selected because it is sufficient in adult zebrafish to completely deplete stored lipid from adipose tissues and reduce body weight and liver size, whereas subsequent refeeding largely restores total adipose tissue lipid, body weight, and liver size within 14 days $37,38,52,53$. We performed 16S rRNA gene sequencing on whole intestinal samples from zebrafish at $0,1,3,7$, and 21 days post-starvation (dpS), then at $1,3,7$, and 21 days post-refeeding (dpR) with a standard diet (Fig. 1A). Age matched siblings fed the same standard diet on a daily basis served as reference controls and were sampled at the same time points.

During these early adult stages, zebrafish fed normally continued to display somatic growth as expected. Measurements of animal size as standard length (SL) and height at anterior anal fin (HAA) revealed that somatic growth in starved fish was largely arrested compared to control fish (Fig. 1BC). Starved fish were significantly smaller than fed fish by 7 days post-starvation and this trend continued beyond the end of the starvation period. Starved animals resumed growth after refeeding, though they remained significantly smaller than fed fish throughout the duration of the experiment $(p<0.05$, twoway ANOVA with Bonferroni correction) (Fig. 1BC). We observed no mortality in any of these conditions consistent with previous studies ${ }^{38,53}$. Starvation therefore caused a general arrest in somatic growth which was restored upon refeeding.

Analysis of 16S rRNA gene sequencing data from intestinal samples revealed the impacts of prolonged starvation and refeeding on intestinal microbiome composition. 
161 diversity compared to fed controls. Both conditions displayed an initial loss of diversity by $1621 \mathrm{dpS}$, perhaps due to stress caused by tank transfer at 0dpS when the experiment began. 163 However starved communities maintained significant higher diversity from $1 \mathrm{dpS}$ through 164 the end of starvation at $21 \mathrm{dpS}$ and again at $21 \mathrm{dpR}(\mathrm{p}<0.05$, ANOVA and Tukey HSD) 165 (Fig. 1D). Beta diversity analysis of community composition using Bray-Curtis distances 166 showed that starved and fed communities began to differ by $1 \mathrm{dpS}$, with the centroid 167 distances being greatest at $3 \mathrm{dpS}$ and $21 \mathrm{dpS}$ (Fig. 2A). During refeeding, the starved fish 168 samples quickly returned to a composition more similar to fed controls (Fig. 2A). 169 PERMANOVA further confirmed that starvation and experimental time point are both 170 significant factors $(p<0.05)$ affecting gut microbiome composition. Thus, prolonged 171 starvation induced detectable shifts in overall composition of gut bacterial communities 172 that were reversed quickly after refeeding. 
Figure 1

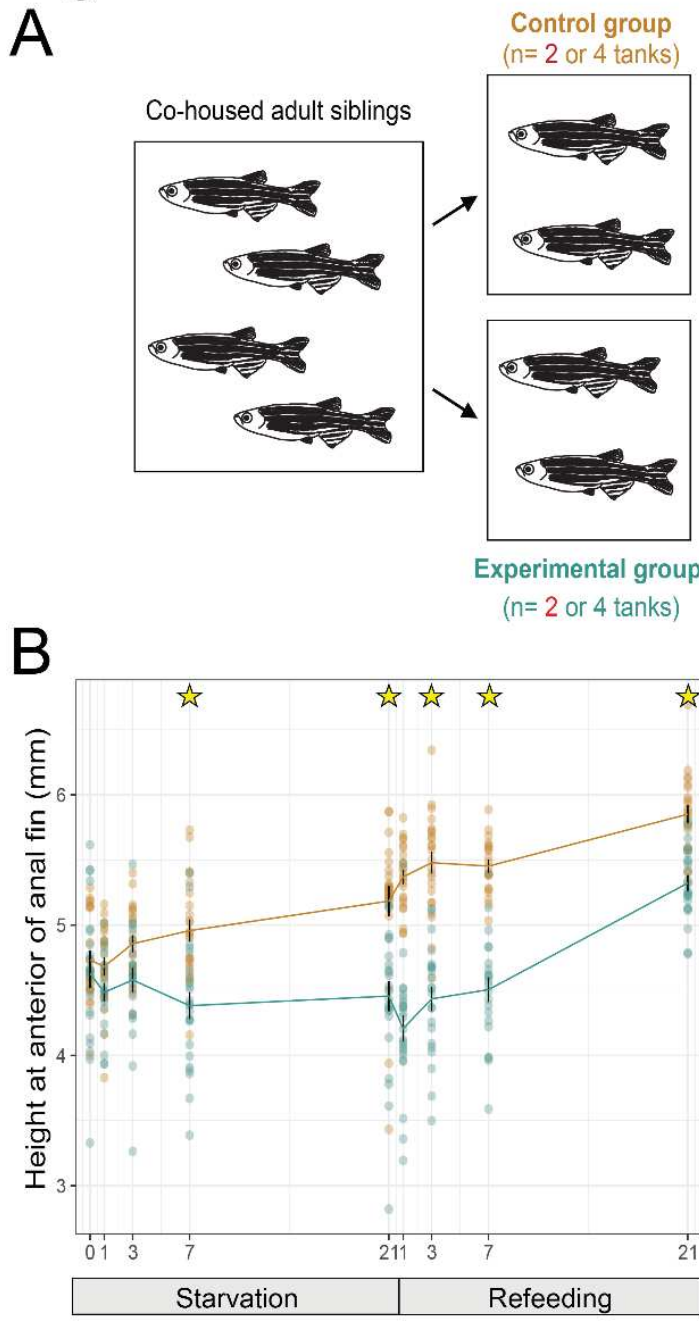

Fed control regimen

$\begin{array}{llllllll}01 & 3^{*} & 7 & 21 & 1 & 3^{*} & 7 & 21^{*}\end{array}$

Days post-starvation (dpS) Days post-refeeding (dpR)

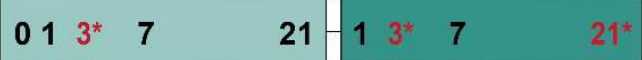

Starvation/Refeeding regimen

*Denotes days RNA-Seq samples were taken

C
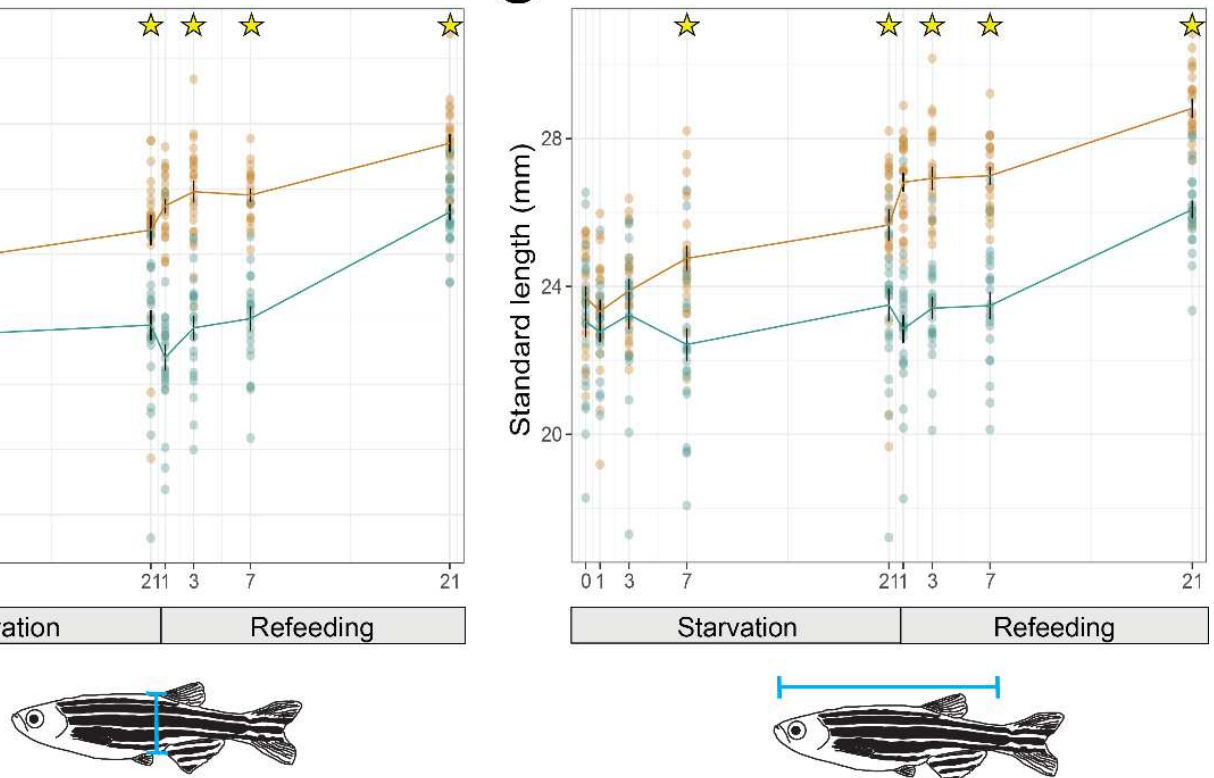

Height at anterior of anal fin

$\mathrm{D}$

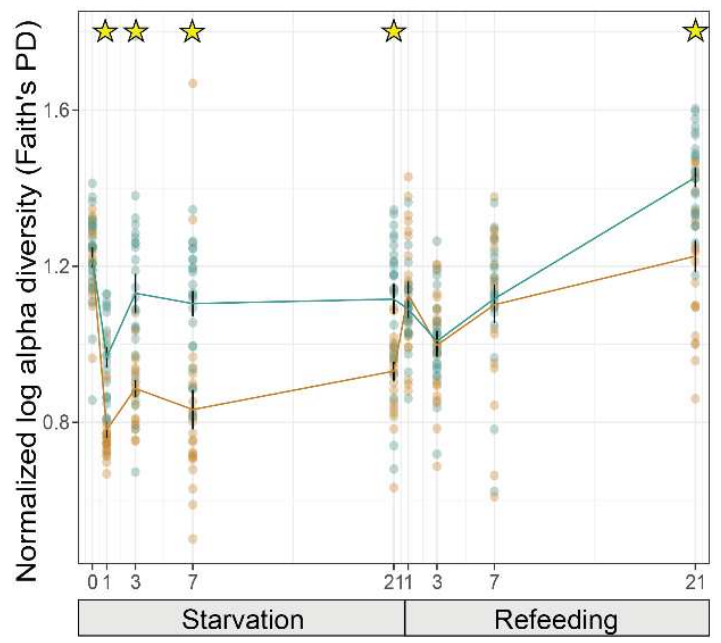

$E$
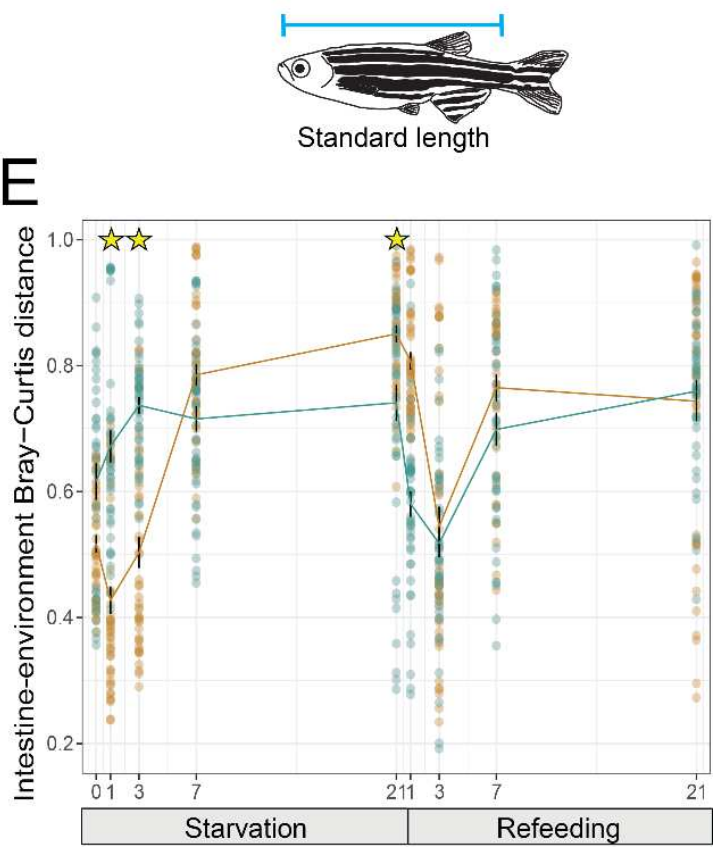
175 Figure 1. Starvation and refeeding affect zebrafish somatic growth as well as intestinal and 176 environmental microbiome diversity.

177 (A) Study design graphical abstract. Cohoused adult siblings were divided into either control (fed) 178 or experimental (starved) tanks. Samples were then taken from each tank on days 0, 1, 3, 7 and 17921 post-starvation (dpS) as well as 1, 3, 7, and 21 days post-refeeding (dpR) for 16S rRNA gene 180 sequencing. RNA-seq samples were taken at $3 \mathrm{dpS}, 21 \mathrm{dpS}$, and $3 \mathrm{dpR}$.

181 (B) Fed and starved zebrafish height at anterior of anal fin (HAA) in $\mathrm{mm}$ at corresponding 182 timepoints.

183 (C) Standard length in mm of starved and fed zebrafish.

184 (D) Faith's PD alpha diversity for fed and control zebrafish. Values are log transformed and 185 normalized by the scores at day 0.

186 (E) Boxplots of the Bray-Curtis distance between the gut and associated environment sample. 187 Stars in panels B-E denote significant difference ( $p<0.05$ by Tukey HSD test). 
Starvation increases similarity between zebrafish gut microbiomes and their surrounding water environment.

The microbiome residing in the zebrafish intestine exists in continuity with that of the surrounding water environment, however these communities typically display distinct compositions ${ }^{42-44}$. The ecological processes contributing to these differences remain unclear, but could include non-neutral processes such as host selection ${ }^{54,55}$ or the magnitude of dispersal between the intestine and the surrounding environment ${ }^{56}$. To test if starvation and refeeding alter the relationship between the gut and environmental microbiomes, we compared Bray-Curtis distance between matched gut and environmental samples in starved/refed and control fish. The distance between gut and environmental samples increased between $0 \mathrm{dpS}$ and $7 \mathrm{dpS}$ (Fig. 1E), perhaps reflecting restoration of homeostasis after the stress of transfer into new tanks that occurred at 0 dpS. However, from 1-3 dpS the distance between gut and environmental samples was greater for starved than fed controls, indicating that the fed controls had an intestinal microbiome composition more similar to the environment compared to starved fish during those stages. By 7-21 dpS, the distance between gut and environmental samples was smaller in starved fish compared to fed controls (Fig. 1E). By $3 \mathrm{dpR}$ and for the remainder of the refeeding period, these distances were not statistically different between treatment groups. These results indicate that starvation has a biphasic effect on the similarity between gut and environmental communities, decreasing similarity during early stages and increasing similarity during later stages. Whereas refeeding rapidly restores differences between these communities to levels achieved under constant feeding conditions.

\section{Vibrio bacteria are significantly enriched in the intestine during starvation}

We next sought to identify the specific bacterial taxa that were significantly affected by starvation and refeeding using LEfSe ${ }^{57}$. LEfSe identified 120 genus-level taxa that reached a logarithmic LDA score of 2.0 (Table S1). This set of affected taxa included twelve abundant genera (median relative abundance $>0.1 \%$ across all samples; shown in Fig. 2B) including starvation-induced depletion of Plesiomonas and enrichment of Vibrio. Strikingly, Vibrio reached a maximum relative abundance of $87 \%$ (median $34 \%$ ) in the intestines of starved zebrafish at $21 \mathrm{dpS}$, which was markedly higher than that of fed controls (maximum 62\%; median 4\%) at the same time point (Fig. 2C). In contrast, starvation led to reduced relative abundance of Plesiomonas in the intestine by $1 \mathrm{dpS}$ continuing through $7 \mathrm{dpR}$ (Fig. 2E). These effects of starvation on Vibrio and Plesiomonas $\mathrm{sp}$. in the starved guts were reflected in the environmental samples of the starved fish (Fig. 2DF). Importantly, none of the phyla or orders that were significantly depleted or enriched were significantly correlated with SL after Bonferroni correction (see Table S2). 
230 This suggests that their depletion and enrichment are due to the dietary treatment, and 231 not simply the growth arrest observed in starved animals (Fig. 1CD). These results 232 establish that prolonged starvation leads to significant alterations in intestinal microbiome 233 composition including marked enrichment of Vibrio genus members, and that these 234 alterations in microbiome composition are largely normalized within 1-3 days of refeeding. 235 Vibrio sp. are common members of the intestinal microbiome in zebrafish, and their 236 relative abundance correlates positively with intestinal inflammation ${ }^{41,42,48}$. This suggests 237 that starvation-induced alterations in the relative abundance of Vibrio sp. and other 238 bacteria in the zebrafish intestine might be linked with alterations in intestinal gene 239 expression. 
Figure 2
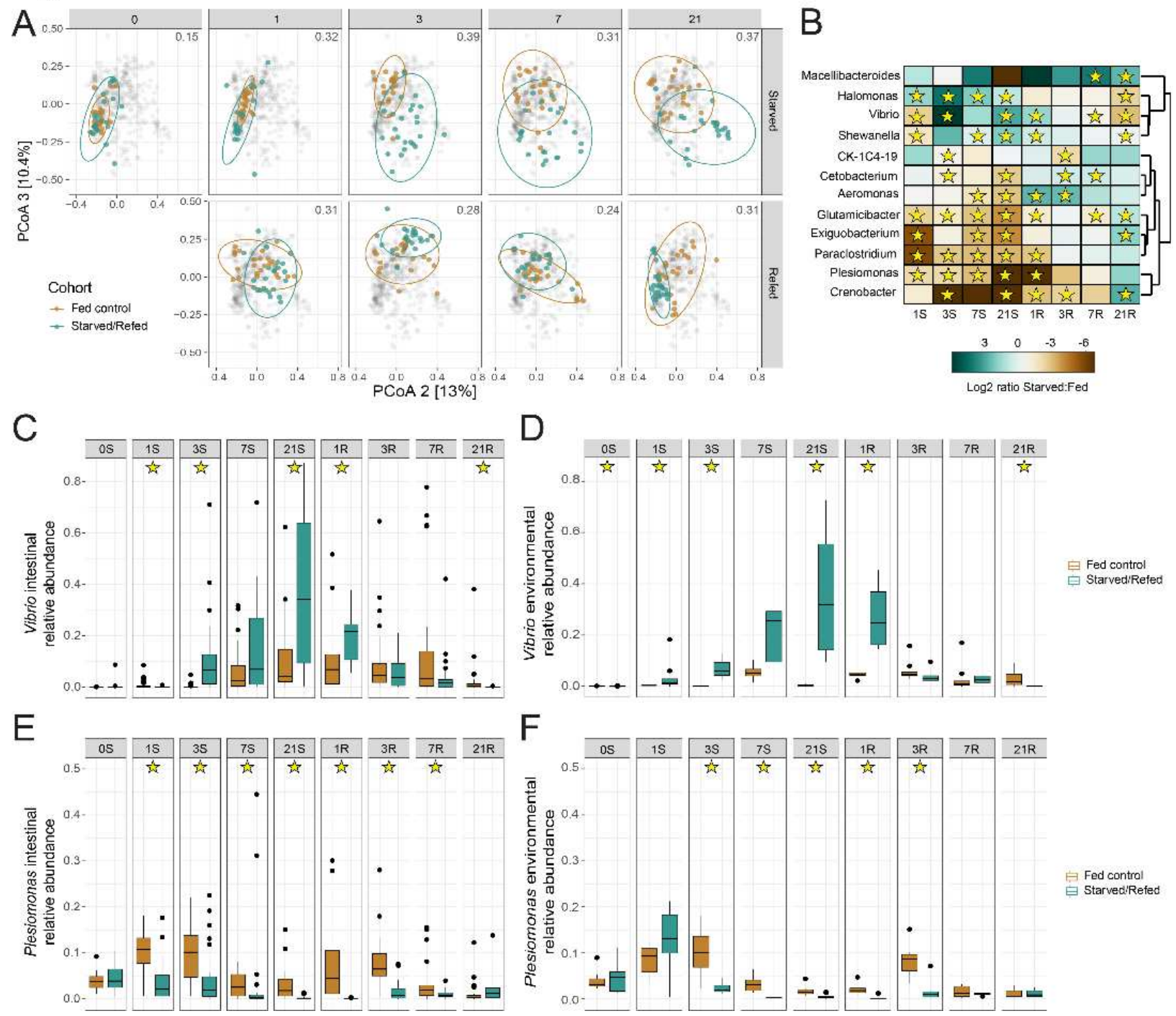

Figure 2. Starvation and refeeding dynamically alters membership of the adult zebrafish intestinal microbiome.

245 (A) Principal coordinates analysis of Bray-Curtis diversity for fed and starved zebrafish. The distance between centroids of the two cohorts at the corresponding timepoint is shown in the top right of each plot.

(B) Heatmap of log2 ratio of the relative abundance of bacterial genera between starved and fed controls. Stars denote day identified as significant by LEfSe.

(C) Relative abundance of Vibrio in starved and control zebrafish intestines by day.

(D) Relative abundance of Vibrio in starved and control environmental tank water samples by day.

(E) Relative abundance of Plesiomonas in starved and control zebrafish intestines by day.

(F) Relative abundance of Plesiomonas in starved and control environmental tank water samples by day. Stars in panels $\mathrm{C}-\mathrm{F}$ denote significance $(p<0.05)$ by pairwise Wilcoxon test with $\mathrm{BH}$ correction. 
Starvation and refeeding leads to distinct changes in intestinal gene expression that vary with the duration of starvation

Previous work in vertebrates has shown that starvation can significantly affect host gene expression in multiple organs $4,7,17,33,52$. However, studies describing transcriptomic changes in the host intestine during starvation are lacking. Our analysis of intestinal microbiomes during starvation and refeeding suggested distinct stages - early starvation when microbiome effects are initially observed (i.e., $3 \mathrm{dpS}$ ), late starvation when microbiome alterations are greatest (i.e., $21 \mathrm{dpS}$ ), and early refeeding when microbiome composition is largely normalized (i.e., $3 \mathrm{dpR}$ ). We sought to evaluate the physiologic status of the intestine at these stages using a transcriptomic approach. Whole intestinal tracts dissected from $3 \mathrm{dpS}, 21 \mathrm{dpS}$, and $3 \mathrm{dpR}$ adult zebrafish and their fed age-matched controls were subjected to RNA-seq analysis (3-4 biological replicate samples/condition; Fig. 1A). Principal coordinates analysis (PCoA) of these data revealed similarities between biological replicates (Fig. 3A). Similar to the observed effects on the intestinal microbiome, the impact of starvation on intestinal gene expression was greater at $21 \mathrm{dpS}$ than $3 \mathrm{dpS}$ or $3 \mathrm{dpR}$. We then used DEseq2 analysis to identify genes differentially expressed in starved/refed fish compared to their fed controls at each timepoint. In accord with our PCoA analysis, the number of significant differentially expressed genes increased from 69 genes at $3 \mathrm{dpS}$ to 167 genes at $21 \mathrm{dpS}$, and was reduced to 11 genes by $3 \mathrm{dpR}$ (Fig. 3B). This further supports that starvation has a progressive impact on the intestinal transcriptome through $3 \mathrm{dpS}$ and $21 \mathrm{dpS}$ which is largely normalized by $3 \mathrm{dpR}$.

As a control and to estimate the influence of the developmental time covered during the experiment, we compared differential gene expression between the fed timepoints. As expected, relatively few genes were found to be differentially expressed between fed timepoints and they were removed from our subsequent analyses of starvation effects (Fig. S1B, Table S3B). Hierarchical clustering of the $\log _{2}$ fold changes in transcript abundance revealed distinct groups of genes that were upregulated or downregulated in response to starvation, including striking differences between the response to starvation at $3 \mathrm{dpS}$ and $21 \mathrm{dpS}$ (Fig. 3C). Though gene expression differences between starved/refed fish and fed controls was largely restored by $3 \mathrm{dpR}$, there was a small set of 11 genes that continued to be differentially expressed even at 3dpR (Fig. 3B; Table S3A). Although this list of persistent genes was too small to permit functional enrichment analysis, it does suggest a potentially small group of intestinal functions that remain altered after restoration of feeding or that respond to both starvation as well as to refeeding. These persistently different genes are discussed below in context.

Among the genes known to be starvation responsive, we first examined transcript levels of elov/2, a fatty acid elongase previously shown to be downregulated in zebrafish during starvation ${ }^{58-60}$. Elovl2 has also been implicated in inducing insulin secretion in response to glucose in mice, and fatty acid elongases have been extensively studied in 
fish as they function in biosynthesis of long-chain polyunsaturated fatty acids, which are commercially important in fish aquaculture $58,61,62$. In accord, elov/2 was significantly downregulated by $3 \mathrm{dpS}$, was one of the most significantly downregulated genes in starved fish at $21 \mathrm{dpS}$, and was also consistently expressed across the control fed fish group (Fig. 3DE, Fig. S1A). This downregulation suggested a reduction in intestinal fatty acid synthesis during starvation.

To understand which biological processes are impacted by starvation, we performed Gene Ontology (GO) term searches of four groups of genes from our dataset; genes significantly upregulated at $3 \mathrm{dpS}$ or $21 \mathrm{dpS}$, and genes significantly downregulated at $3 \mathrm{dpS}$ or $21 \mathrm{dpS}$ (Figs. 3C, S1CD, and S2). We first identified distinct, non-overlapping functions that were enriched early in starvation (i.e., at 3dpS) and late in starvation (i.e., not $21 \mathrm{dpS}$, included "ribosome" and "ribosome large subunit biogenesis" (Fig. S1E). These included the ribosome biogenesis factor nsa2 and gtpbp4 which is involved in biogenesis of the $60 \mathrm{~S}$ ribosomal subunit, which were significantly upregulated at $3 \mathrm{dpS}$ but not $21 \mathrm{dpS}$. However, the function "ribosomal large subunit assembly" was enriched among genes upregulated at $21 \mathrm{dpS}$ and not $3 \mathrm{dpS}$. This included some genes that were only significantly increased at $21 \mathrm{dpS}$ such as ruvb/1 and srfbp 1 , and others that were significantly increased at both $3 \mathrm{dpS}$ and $21 \mathrm{dpS}$ such as $r$ s/24d1, ptges $3 /$, and gltscr2 (Table S3A). Overall, genes involved in ribosome biogenesis were induced more strongly at $3 \mathrm{dpS}$ compared to $21 \mathrm{dpS}$, suggesting it is a relatively early response to starvation with aspects that continue through $21 \mathrm{dpS}$ (Fig. S2CD, Table S3A).

Also among the genes upregulated specifically at $3 \mathrm{dpS}$ was the heat shock protein hsp90ab1, a molecular chaperone previously shown to be upregulated in adult zebrafish liver in response to starvation ${ }^{17}$. The most significantly upregulated gene in our dataset was the enteropeptidase/enterokinase tmprss15, that converts trypsinogen into active trypsin which in turn activates pancreatic enzymes and potentially also antimicrobial proteins in the intestinal lumen (Fig 3DF) ${ }^{63,64}$. Intestinal expression of tmprss 15 was not affected by starvation at $3 \mathrm{dpS}$, but was upregulated 6-fold by $21 \mathrm{dpS}$ (Fig. 3DF). Notably, a deficiency in TMPRSS15 has been shown to confer a lean, starvation-like phenotype in humans, consistent with its known key role in nutrient digestion and absorption ${ }^{65}$. Upregulation of tmprss15 in the starved zebrafish intestine suggests potential adaptive increases in nutrient digestion programs to salvage nutrients from the intestinal lumen, or in antimicrobial defense against an altered and potentially pro-inflammatory microbiome.

Similarly, functions enriched specifically among downregulated genes at 3dpS and not at $21 \mathrm{dpS}$ included "metabolism of lipids", "regulation of cell proliferation", and "ubiquitin-dependent protein catabolic process" (Fig. S1E). This included downregulation of the acyltransferase Iclat 1 and fatty acid binding protein fabp $1 \mathrm{~b} .1$ at $3 \mathrm{dpS}$ but not $21 \mathrm{dpS}$. However, related functions "glycerophospholipid metabolism", "lipid transport", and "lipid metabolic process" were enriched among downregulated genes at $21 \mathrm{dpS}$ but not $3 \mathrm{dpS}$. 
337 These included the phospholipase pla2g12b which regulates lipoprotein size, the fatty 338 acid desaturase fads2, the lipid transfer protein scp2a, and multiple apolipoproteins 339 including apoa1a, apoa4b.1, and apobb.1 (Figs 3D, S1E, S2AB) 66. Notably, 340 apolipoprotein genes have been shown to be downregulated in starved rainbow trout livers ${ }^{67}$. Although these genes were significantly downregulated only at $21 \mathrm{dpS}$, most began trending towards downregulation at $3 \mathrm{dpS}$. Yet other genes involved in these functions including elovl2 and fabp $1 b .1$ were significantly downregulated at both $3 \mathrm{dpS}$ and $21 \mathrm{dpS}$ with a larger difference at $21 \mathrm{dpS}$ (Figs. 3D and S1A, Table S3A). Notably, the transporter s/c31a1/ctr1 involved in dietary copper uptake was also significantly downregulated at both timepoints. Thus, while shorter durations of starvation such as 3 days lead to a downregulation of some metabolic functions, most of the genes involved in lipid metabolism are not significantly downregulated until 21 days of starvation. Prolonged starvation therefore leads to reduced expression of genes involved in lipid biosynthesis and transport, perhaps representing an adaptation to the prolonged absence of dietary fats and other nutrients. However, this contrasts with shorter periods of starvation, such as 48 hours, where other zebrafish studies have observed an increase in lipid catabolism, potentially to increase available energy and improve resistance to cold 68 .

The genes significantly downregulated by starvation were also enriched for host immune functions. For example, the signal transducer stat $1 b$, which is required for inflammatory responses in the intestine and for myeloid development in zebrafish ${ }^{69,70}$, and the interferon responsive gene ifit8 are downregulated by $3 \mathrm{dpS}$ and continuing through $21 \mathrm{dpS}$. By $21 \mathrm{dpS}$, ifit 15 and the antiviral protein rsad2 are also significantly downregulated. In accord, downregulated genes at $21 \mathrm{dpS}$ were enriched for functions involved in "defense response to virus". Finally, the carboxypeptidase cpa5 which is a marker for mast cells in zebrafish ${ }^{71}$ was also significantly downregulated at $21 \mathrm{dpS}$, suggesting a potential reduction in mast cell number or activity within the intestinal tissue. Although several immune-related genes were downregulated in starved fish, complement proteins $c 6$ and $c 7$ were both significantly upregulated in starved fish (Table S3). Our analysis of genes downregulated during starvation therefore suggests a reduction or impairment in immune function and inflammatory tone in the intestine during starvation, along with significant reductions in lipid metabolism and lipoprotein production. Reduced immune function during starvation may represent a mechanism contributing to the microbial community alterations observed at those timepoints.

While there were too few significant genes after refeeding at $3 \mathrm{dpR}$ to permit analysis of functional enrichment, several of these genes were suggestive of potential intestinal functions. This included increased expression at $3 \mathrm{dpR}$ of genes encoding the tandem-duplicated trypsin-like serine proteases prss59.1 and prss59.2. This small set of genes also included three mitochondrial enzymes beta carotene dioxygenase-like gene bco2l, involved in cleavage of dietary carotenoids into retinoids towards Vitamin A 
synthesis; and dimethylglycine dehydrogenase $d m g d h$, involved in glycine synthesis and production of sarcosine in the choline oxidation pathway. Notably, Dmgdh was previously shown to be induced in mouse livers upon fasting, and reduced in the livers of ground squirrels preparing for hibernation ${ }^{72,73}$. Of the 11 genes differentially expressed in $3 \mathrm{dpR}$ refed fish compared to fed controls, 6 were also differentially expressed at $21 \mathrm{dpS}$ including prss59.1, prss59.2, and bco2l. These may represent starvation adaptations that remain altered after restoration of feeding or that respond to both starvation as well as to refeeding.

Although we had already removed from this analysis any genes that were differentially expressed between fed control timepoints (Table S1B), we wanted to further evaluate whether there were broader biological processes that may have been differential between those fed control samples that could affect our comparisons with starved/refed animals. We therefore performed GO term analysis of genes identified as significantly different between our fed control timepoints. The GO term "lipid metabolic process" was significantly enriched among genes that were significantly downregulated in $21 \mathrm{dpS}$ fed relative to $3 \mathrm{dpS}$ fed fish. Conversely, the GO terms "lipid localization" and "response to lipid" were significantly enriched among genes that were significantly upregulated in $21 \mathrm{dpS}$ fed relative to $3 \mathrm{dpS}$ fed fish (Table S3D). Importantly, the GO term "lipid metabolic process" was also enriched in genes that were significantly downregulated in $21 \mathrm{dpS}$ starved relative to $21 \mathrm{dpS}$ fed fish, even after genes that were significant in our control analysis were removed (Fig. S1E). This raised the possibility that our observed impacts of starvation on lipid metabolism genes here may be driven in part by unusually low expression of certain lipid metabolic genes in $21 \mathrm{dpS}$ fed fish, whereas other related lipid metabolic functions may be unusually high in $21 \mathrm{dpS}$ fed fish relative to the other fed timepoints. We therefore evaluated the log2 fold changes of genes from this control analysis alongside genes that were significantly different between starved and fed fish to discern if some of these differences may be driven by the control 21dpS fed fish (Fig. S2). We found that genes under the GO terms "ribosome" and "ribosome large subunit biogenesis" do not have differential expression in starved fish that is affected by unusual gene expression in the fed fish (Fig. S2CD). In contrast, a subset of genes such as $p d k 3 b$, syt1b, apoa1a, apoa $4 b .1$, and fads 2 which are significantly downregulated in starved fish at $21 \mathrm{dpS}$ relative to $21 \mathrm{dpS}$ fed, may be due in part to unusually high expression of these genes in 21dpS fed fish (Fig. S2AB). However, most genes emphasized here, such as elov/2, pla2g12b, s/c31a1, and many others, are not affected by abnormalities within the fed fish cohort and are likely true biological effects of the starvation treatment. 
Figure 3

A

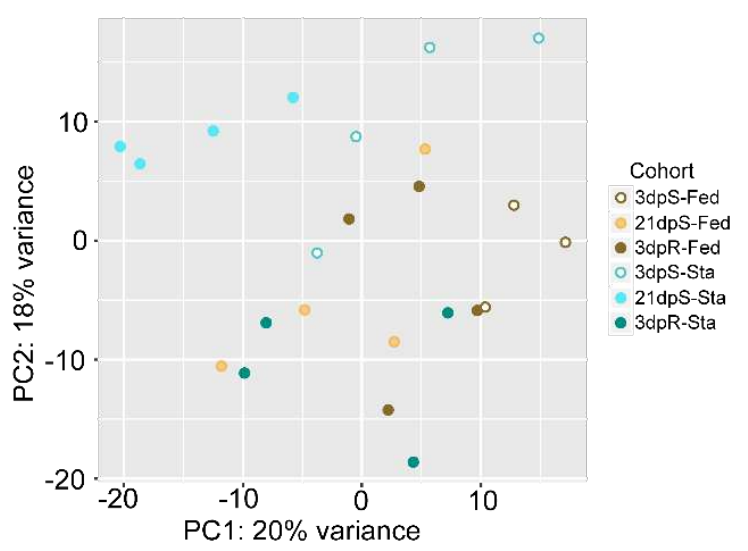

B

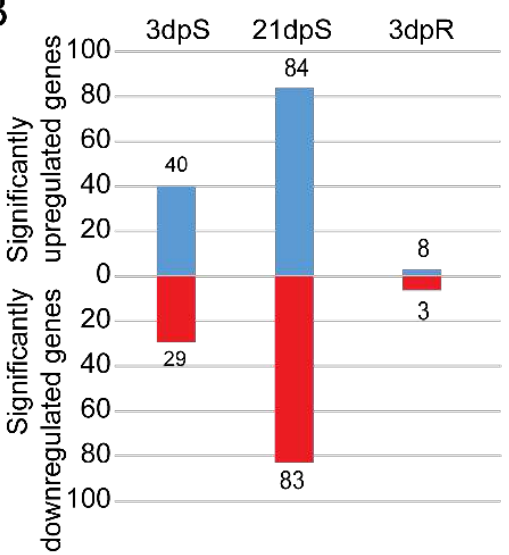

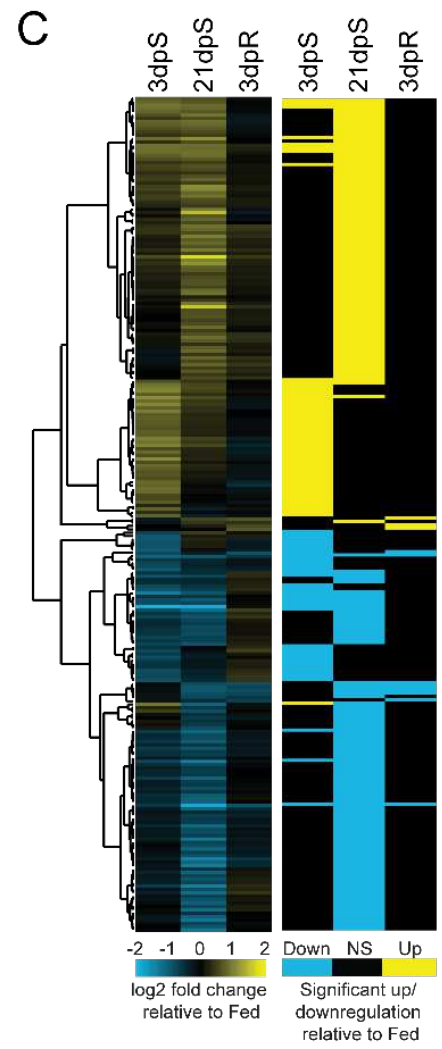

E

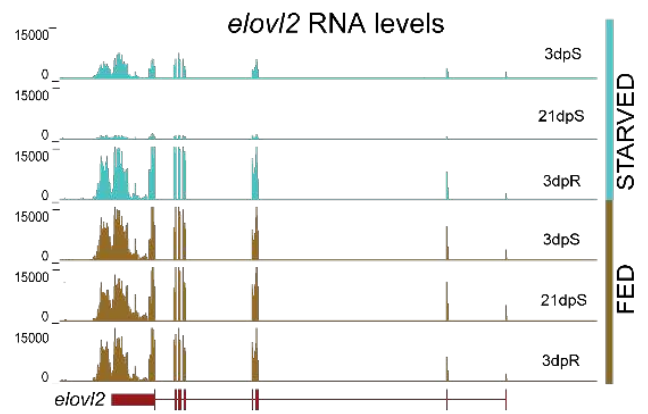

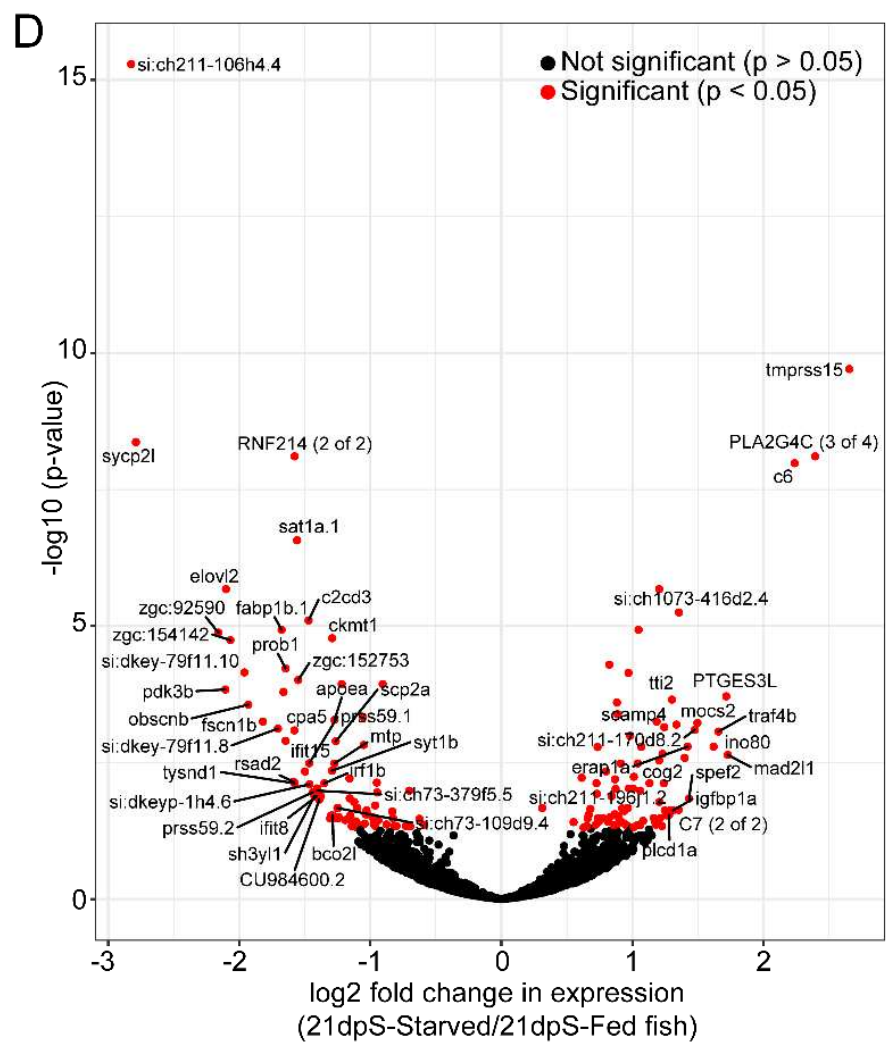

F

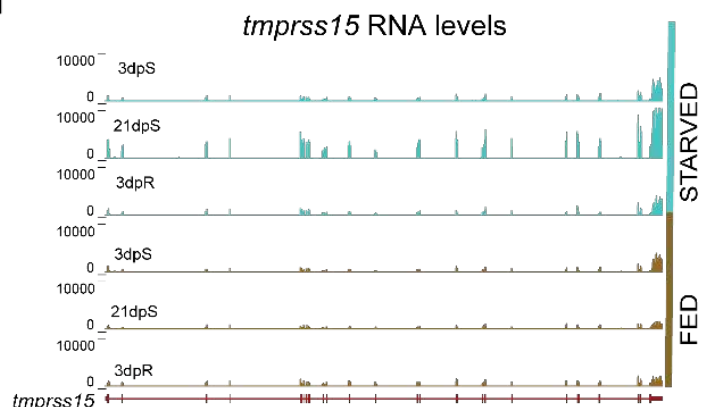


414 Figure 3. Starved zebrafish differentially regulate intestinal gene expression when 415 compared to fed zebrafish

416 (A) PCoA analysis of RNA-Seq libraries in starved/refed and fed control zebrafish intestines at 417 3dps, 21dps, and 3dpR.

418 (B) Quantification of the number of significantly upregulated and downregulated genes in 419 starved/refed zebrafish intestines at each timepoint. Note that these numbers reflect totals after 420 removing genes that were also significantly changed in our fed control comparisons.

421 (C) Hierarchical clustering of log2 fold changes in gene expression in starved zebrafish intestines, 422 along with flattened values that show significant changes in gene expression.

423 (D) Log2 fold changes in gene expression in starved zebrafish intestines at $21 \mathrm{dpS}$ when 424 compared to $21 \mathrm{dpS}$ fed fish plotted according to their - $\log _{10}$ adjusted $p$-values.

425 (E) UCSC tracks of representative replicates show that elovl2 mRNA, encoding a fatty acid 426 elongase, is downregulated in starved zebrafish intestines and returns to levels comparable to 427 the fed group upon re-feeding.

428 (F) UCSC tracks of representative replicates show that tmprss15 mRNA, encoding an 429 enteropeptidase, is upregulated in starved zebrafish intestines and returns to levels comparable 430 to the fed group upon re-feeding. 
To interpret which starvation-responsive genes from our dataset responded transcriptionally across a broad range of nutrient availability, and which ones may constitute a starvation-specific response, we referenced our intestinal RNA-seq results against previously published RNA-seq data comparing digestive tracts from zebrafish larvae that were either unfed or fed a high-fat meal (chicken egg yolk) ${ }^{59}$. This revealed a large overlap in significantly differentially-expressed genes (Fig. 4). Particularly, genes involved in lipid transport and metabolism such as fabp1b.1 and pla2g12b that were downregulated during starvation were upregulated during high fat feeding in zebrafish, underscoring the ability of these genes to respond to nutrients in zebrafish. Several genes involved in immune function such as rsad2, stat $1 b$, and ifit 15 were downregulated during starvation, and were upregulated after high fat feeding. Also among the overlapping genes was the enteropeptidase tmprss 15 , which was upregulated during starvation but downregulated by high fat feeding.

While there was an overlap between genes in the above datasets that implicated them in the intestinal nutrient response, several genes that were significantly affected by starvation were not significantly affected by high fat feeding. These genes included the complement factor $c 6$, the fatty acid elongase elov/2, and the phospholipase pla2g4c. These findings suggest that some classes of genes involved in lipid transport or inflammation may be differentially regulated by factors uniquely associated with starvation and not nutrient excess inherent to high fat feeding. Alternatively, these differences could be ascribed to transcriptional responses unique to zebrafish life stages (adult vs larvae) or organs (intestine vs complete digestive tract including intestine, liver, pancreas, and swim bladder), or to indirect effects of high fat egg yolk feeding that are unrelated to nutrition. 


\section{Figure 4}
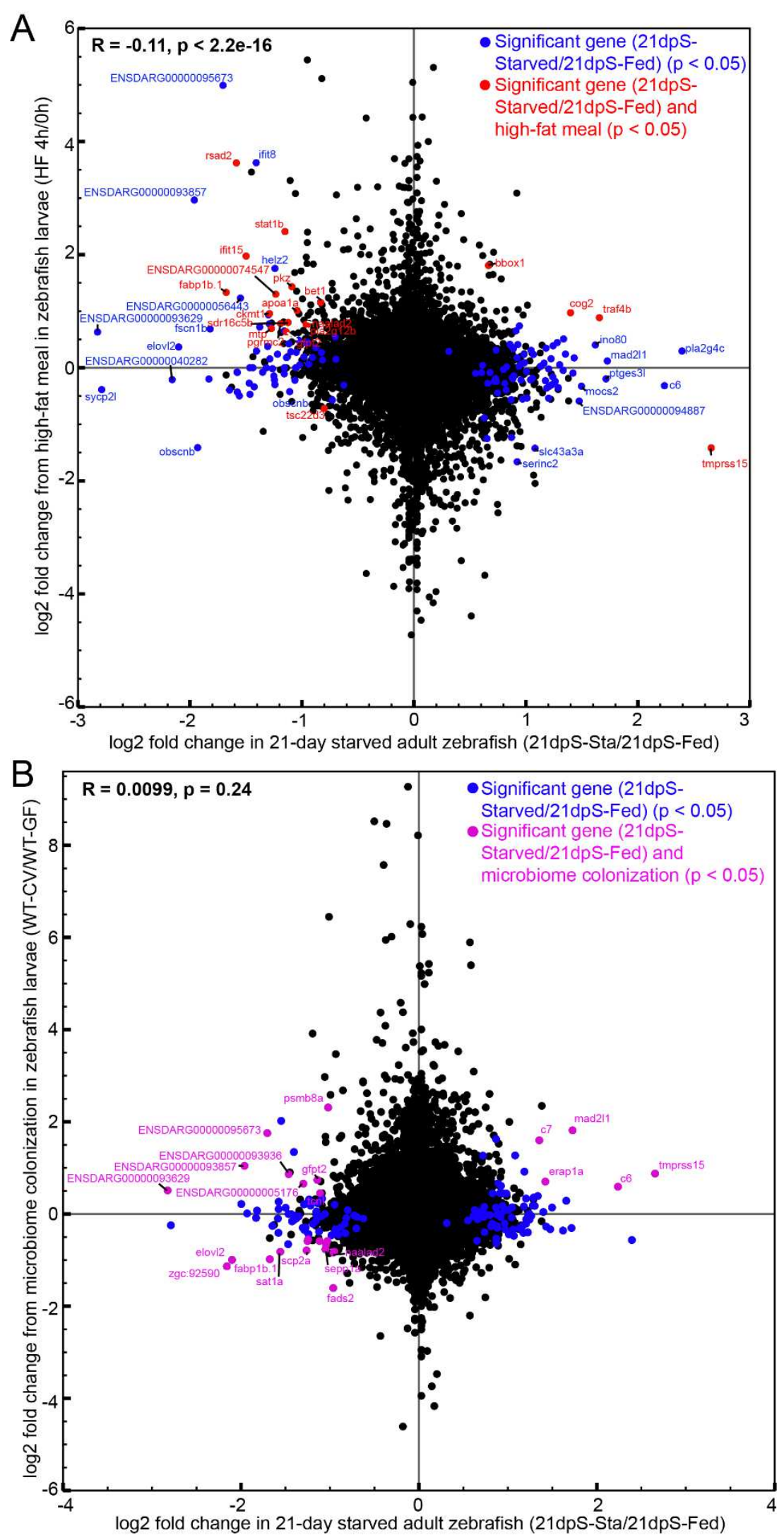
462 Figure 4. Some genes responsive to starvation in the intestine are also responsive to high 463 fat feeding and microbial colonization

464 (A) Log2 fold changes for genes from 21dpS (X-axis) plotted according to their log2 fold changes 465 in egg yolk-fed larval zebrafish compared to unfed controls (Y-axis), described in Zeituni et al ${ }^{59}$. 466 Significantly differential genes only in starved zebrafish are plotted in blue, whereas genes 467 significant in both datasets are plotted in red. Pearson's correlation revealed a significant 468 correlation between the two datasets $(p<0.05)$.

469 (B) Log2 fold changes for genes from 21dpS (X-axis) plotted according to their log2 fold changes 470 in zebrafish larvae colonized with a microbiome compared to germ-free controls (Y-axis), 471 described in Davison et al ${ }^{75}$. Genes with significant log2 fold changes only in starved zebrafish 472 are plotted in blue, whereas genes significant in both datasets are plotted in magenta. Pearson's 473 correlation did not reveal a significant correlation between the two datasets $(p>0.05)$. 
A small subset of genes responsive to starvation are also responsive to microbial colonization

We and others have shown that intestinal gene expression is regulated in part by the presence and composition of the intestinal microbiome ${ }^{55,74-76}$. Our 16S rRNA sequence data revealed that starvation induced marked and reversible alterations to gut microbiome composition including enrichment of Vibrio sp., members of which have been shown to be pro-inflammatory, and a decrease in similarity in microbiome composition between starved fish and their environmental samples ${ }^{48}$. Although these results suggest altered gut microbial ecology during starvation, our study design did not permit us to causally link our observed changes in intestinal transcriptome and microbiome. Therefore, in order to identify transcriptional responses to starvation that may also be sensitive to microbiome, we compared our RNA-seq data to a previous study investigating the effect of microbial colonization on larval zebrafish digestive tracts ${ }^{75}$. We found a relatively low correlation between the two datasets, implying that there may not be extensive overlaps between transcriptional responses to microbial colonization and starvation at these timepoints (Fig. 4B). This modest overlap may be due to transcriptional responses unique to zebrafish life stages (adult vs larvae) or organs (intestine vs complete digestive tract including intestine, liver, pancreas, and swim bladder). However, we did identify several overlapping genes that were significant in both datasets. Two complement factors, $c 6$ and $c 7$, which were downregulated in germ-free zebrafish, were upregulated during starvation. In addition, two genes involved in the processing of major histocompatibility complex, mad2l and erap1, were also among the overlapping genes. A small set of genes involved in lipid metabolism and intracellular cholesterol transport, such as fabp $1 b .1$ and scp2a were significantly downregulated in both datasets. The enteropeptidase tmprss 15 , which was differentially expressed during both starvation and high fat feeding, was also significantly downregulated in germ-free zebrafish. Overall, this comparison identified candidate genes that respond to both starvation and microbiome induced pathways. 


\section{Figure 5}

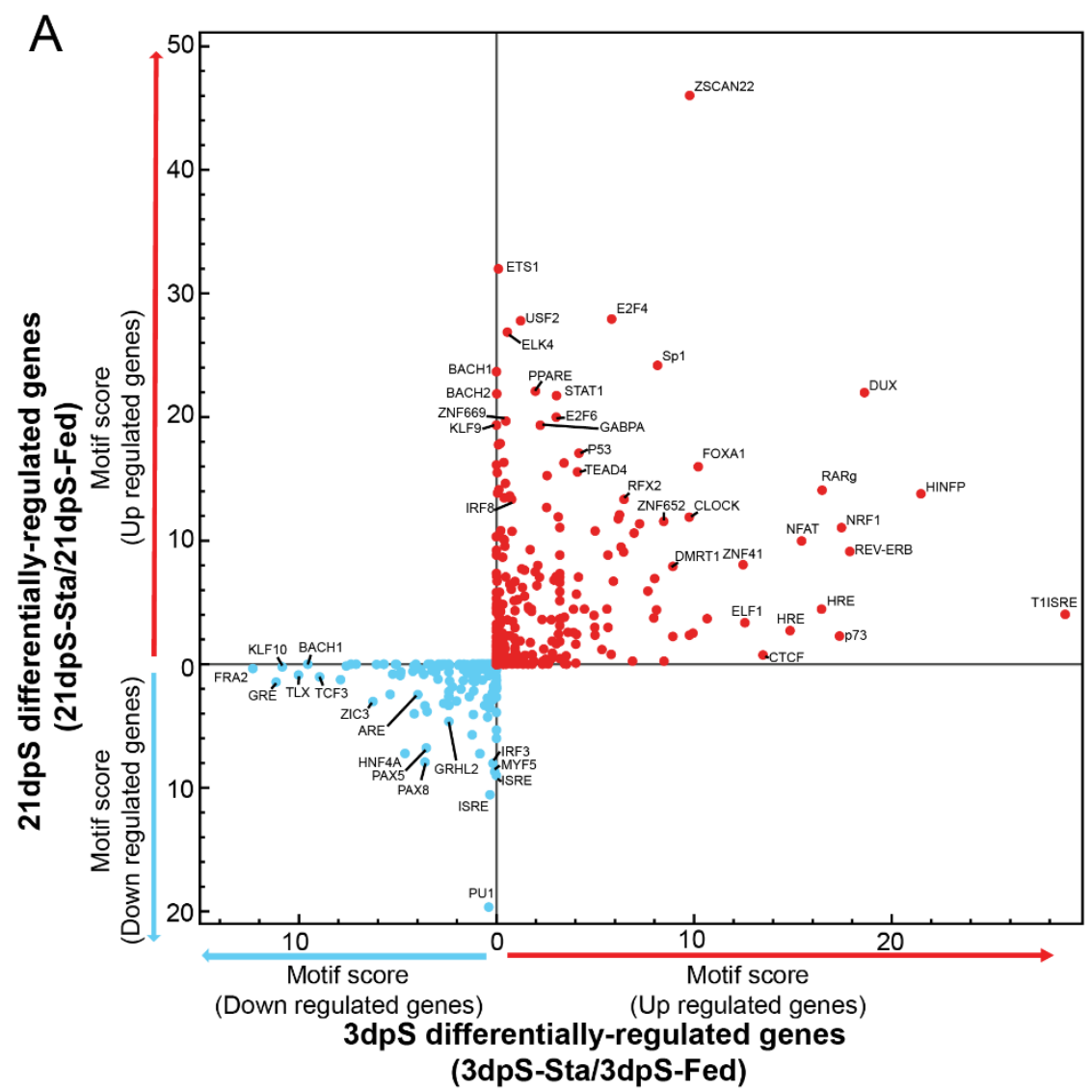

B

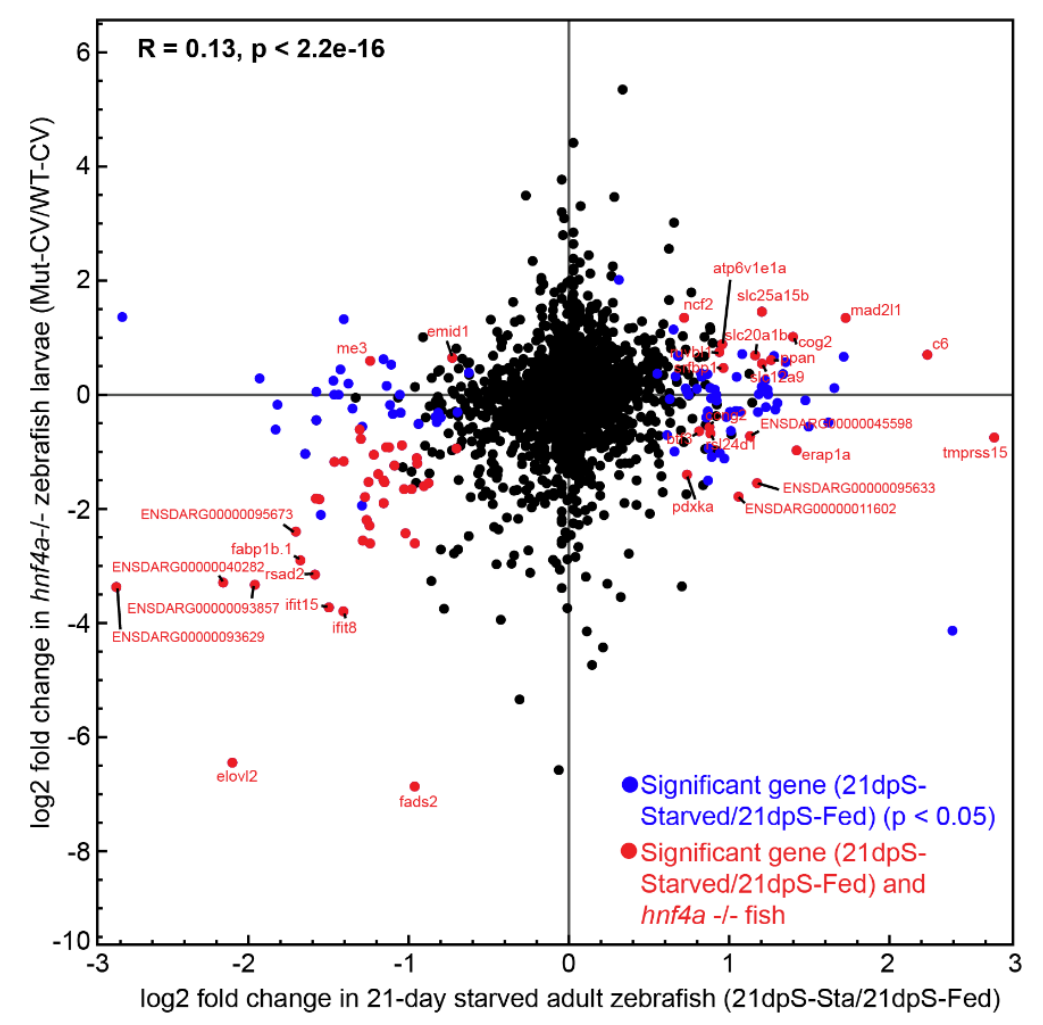


508 Figure 5. The transcription factor hnf4a may regulate a subset of genes involved in 509 starvation

510 (A) Scatterplot for motif enrichment scores for genes at 3dpS (X-axis) and motif enrichment scores 511 for genes at 21dpS (Y-axis), according to HOMER analysis of transcription factor binding sites 512 within $10 \mathrm{~KB}$ upstream or downstream of the genes' transcription start sites at each time point, 513 based on whether these sites were located within accessible chromatic regions. $H N F 4 A$ is among 514 the transcription factors whose binding sites are enriched at genes downregulated at both $3 \mathrm{dpS}$ 515 and $21 \mathrm{dpS}$.

516 (B) Log2 fold changes for genes from 21dpS (X-axis) plotted according to their log2 fold changes 517 in digestive tracts dissected from hnf4a mutant zebrafish larvae compared to wild-type controls 518 (Mut-CV/WT-CV) (Y-axis), described in Davison et al ${ }^{75}$. Genes with genes with significant 519 differential gene expression (21dpsSta/Fed) changes only in starved zebrafish are plotted in blue, 520 whereas genes significant in both datasets are plotted in red. Pearson's correlation revealed a 521 significant correlation between the two datasets $(p<0.05)$. 
Starvation-responsive genes may also be controlled by the transcription factor Hepatocyte nuclear factor 4 alpha (HNF4A)

We next sought to identify transcription factors putatively linked to the regulation of the starvation response. Using HOMER ${ }^{77}$, we queried the genomic regions near all genes identified as significantly upregulated or downregulated by starvation. We restricted our search to regions within the gene body plus the flanking $10 \mathrm{~kb}$ upstream and downstream that we previously identified as accessible chromatin in the zebrafish intestine ${ }^{51}$. This revealed vertebrate transcription factor motifs significantly enriched near starvation responsive genes at either 3dpS or $21 \mathrm{dpS}$ (Fig. 5A; Table S3C). PAX5 motifs were significantly enriched near downregulated genes, while FOXA1 motifs were significantly enriched near upregulated genes, both at 3dpS and 21dpS. Both PAX5 and FOXA1 have been implicated in intestinal development in mice ${ }^{78,79}$. Further, HNF4A motifs were significantly enriched near downregulated genes at both $3 \mathrm{dpS}$ and $21 \mathrm{dpS}$. We previously showed that the nuclear receptor HNF4A mediates host transcriptional responses to microbial colonization in zebrafish ${ }^{75}$. In addition, HNF4A is required for intestine-specific gene regulation and has conserved roles in glucose homeostasis, gluconeogenesis, and lipid metabolism, indicating that starvation-linked genes may be under the control of HNF4A ${ }^{80-83}$. This observation suggested that HNF4 transcription factors might facilitate both responses to starvation and changes to host microbiome.

Based on these previous findings, a comparison of our starvation dataset to an

Interaction between the microbiome and host metabolism is known to occur in diverse pathophysiological contexts including starvation and malnutrition. However, few previous studies have simultaneously explored changes in host gene expression and microbiome RNA-Seq dataset from hnf4a mutant zebrafish ${ }^{75}$ demonstrated that genes putatively controlled by hnf4a were significantly downregulated in our dataset (Fig. 5B). GO Term analysis revealed that "lipid metabolic process", "viral response", and a variety of other metabolic functions were significantly enriched among these overlapping genes. Specifically, the genes pla2g12b and elov/2, and other genes involved in lipid metabolism that were downregulated significantly in starved fish, were all downregulated in $\mathrm{hnf} 4 \mathrm{a}^{-/}$ fish, implying that lipid metabolic responses to starvation might be positively regulated by Hnf4a. Similarly, several of the immune response genes downregulated in starved fish such as ifit15, ifit8, c6, and erap1a were differentially regulated in $h n f 4 a-/-$ fish, suggesting that the immune response to starvation may also be partly influenced by Hnf4a function.

\section{Discussion}

composition as a function of starvation ${ }^{84}$. We focused here on the intestine as the animal's primary interface with the gut microbiome and dietary nutrients. Our RNA-Seq 
data suggests that cytoplasmic translation, ribosomal genes, and ribosomal synthesis genes are upregulated in the zebrafish intestine early in starvation, whereas DNA repair, and vitamin and cofactor metabolism genes become upregulated at $21 \mathrm{dpS}$. Similarly, some pathways significantly downregulated at $21 \mathrm{dpS}$ were distinct from those downregulated at $3 \mathrm{dpS}$, with $21 \mathrm{dpS}$ including genes involved in antiviral response, arginine and proline metabolism, and glycerophospholipid metabolism, among others. The distinct functions encoded at $3 \mathrm{dpS}$ and $21 \mathrm{dpS}$ suggest different stages of starvation, as previously described in zebrafish liver ${ }^{52}$. In sharp contrast to previous studies in other organs, only a handful of genes were differentially expressed after refeeding in the zebrafish intestine, suggesting that the adaptive physiology displayed by the intestine during prolonged starvation is rapidly reversible after refeeding. In starved and refed zebrafish livers, upregulated genes are enriched for functions such as the TCA cycle, proteasome assembly, oxidative phosphorylation, and DNA replication and repair ${ }^{52}$. Similar compensatory mechanisms have been observed to accompany refeeding in cattle livers, as well as in salmon and trout muscle ${ }^{85-87}$. These results suggest that the intestine may be particularly plastic in its adaptation to starvation and refeeding compared to other organs such as muscle and liver.

To explore gene regulatory mechanisms underlying the intestinal response to starvation, we provide evidence that hnf4a may regulate a substantial number of these starvation-associated changes, expanding the already large number of physiologic functions associated with this gene. Considering that $h n f 4 a$ activity is suppressed by the microbiome in zebrafish and mice ${ }^{75}$, hnf4a may link alterations in the host microbiome and transcriptome during starvation. Future studies could test the impact of starvation on Hnf4a occupancy using ChIP-Seq, or on chromatin accessibility or histone modifications in the intestinal epithelium to identify cis-regulatory regions involved in coordinating the starvation response. Our data also provide numerous candidate genes that can be used in future experiments to explore the specificity, regionality, and regulation of the starvation responses in the zebrafish intestine.

Although our RNA-Seq data suggests many commonalities between the starvation response in zebrafish and other vertebrates, it also highlights unique ways in which the zebrafish intestine may adapt to long-term starvation. For example, we observed an induction of complement proteins, ribosomal proteins, and a downregulation of the antiviral response during starvation. This is in contrast to rainbow trout liver where starvation was reported to reduce expression of ribosomal proteins ${ }^{67}$. Meanwhile, genes significantly downregulated at $21 \mathrm{dpS}$ included several involved in the antiviral response. These pathways have not been previously reported in other animals in the context of starvation and thus could represent adaptive mechanisms unique to the zebrafish.

Starved zebrafish exhibited significantly reduced growth that was not fully recovered during a 21-day refeeding timeline. This suggests that full somatic recovery from starvation may require more time, or that there are permanent somatic changes 
associated with starvation. In contrast, we find that the changes that starvation induces in the zebrafish intestinal transcriptome and microbiome are rapidly normalized after refeeding. Whereas starvation significantly affected the expression of over 200 genes in the intestine compared to fed controls, refeeding for just 3 days restored normal levels of expression for all but 11 genes (Fig. 3BC). Similarly, intestinal microbial communities subjected to starvation displayed significantly increased diversity (Fig. 1D) and altered composition (Fig. 2A) compared to fed controls, yet those differences were largely normalized within 1 to 3 days of refeeding. By comparison, another animal that undergoes prolonged starvation, the hibernating ground squirrel, maintained baseline levels of intestinal microbiome diversity during early stages of winter hibernation, reduced diversity later in the winter, and then increased diversity upon refeeding in the spring ${ }^{88}$. That boost in diversity upon refeeding was attributed to new bacterial taxa associated with the introduced food. For this study, no samples of food-associated bacterial taxa were taken, so we cannot distinguish between these two possible explanations. Regardless, the distinct effects of starvation and refeeding on intestinal microbiome diversity in zebrafish and ground squirrels underscores the importance of studying the ecology and physiology of prolonged starvation and refeeding in diverse animal hosts.

Our results also provide insight into the specific bacterial lineages that are most senstivie to starvation and refeeding in the zebrafish intestine. We previously demonstrated that Vibrio and Plesiomonas genera are part of a core gut microbiome of zebrafish ${ }^{89}$. We speculate that the opposing changes in relative abundance of these two taxa likely reflect differing abilities to survive in the altered environment of the starved gut (Fig. 2BCE). During starvation in chickens, intestinal mucus is known to increase in abundance and thickness, possibly creating a competitive advantage for mucindegrading bacteria ${ }^{90,91}$. Vibrio spp. can degrade intestinal mucus, which may be why there is an observed increase in Vibrio during starvation ${ }^{92,93}$. Conversely, Plesiomonas may be less suited for survival during prolonged starvation periods within the gut. It remains unclear if these changes in relative abundance were accompanied by alterations in microbial community density, which could be explored in future studies. It is striking that these and other starvation-induced perturbations to gut microbiome composition, similar to host gene expression in the gut, were largely restored within 1 to 3 days after refeeding. This underscores remarkable plasticity in intestinal physiology and microbial ecology in response to starvation and refeeding.

\section{Methods}

\section{Animal husbandry}

Unless otherwise stated, all fish were maintained on a 14-hour light cycle at $28^{\circ} \mathrm{C}$. All zebrafish used for were born on the same day from 1 (RNA-Seq) or 3 (16S rRNA gene 
644 amplicon sequencing) breeding pairs from a single sibship. Fertilized embryos were 645 transferred into Petri dishes containing egg water ( $6 \mathrm{~g}$ sea salt, $1.5 \mathrm{~g}$ calcium sulfate, 0.75 646 g sodium bicarbonate, 10-12 drops methylene blue, 10L water) at a density of 50 647 embryos/dish at incubated at $28.5^{\circ} \mathrm{C}$. At 1-day post-fertilization (dpf), embryos were 648 transferred to $3 \mathrm{~L}$ tanks containing $500 \mathrm{~mL}$ water from a recirculating zebrafish aquaculture 649 system (system water). Each tank contained 10 (16S rRNA gene sequencing) or 30 650 (RNA-Seq) embryos. Fish were then maintained under standard zebrafish husbandry until 651 the start of the experiment at $60 \mathrm{dpf}$. Zebrafish were then randomly transferred into four 652 (RNA-Seq) or eight (16S rRNA gene sequencing) clean 10L tanks at a density of 44 653 (RNA-Seq) or 67 (16S rRNA gene sequencing) fish per tank, with half the tanks receiving 654 no food for the following 21 days (Fig. 1A). Following the 21 days of starvation, feedings 655 for all tanks were allowed to occur as per standard husbandry: two feedings of Artemia 656 per day interspersed with two feedings of Gemma 300 (Skretting). Over the 21 days of starvation and 21 days of refeeding, we observed no mortality in any condition or 658 experiment.

All fish to be sampled on a particular day were collected prior to the first daily feeding in the fish facility. Samples for $16 \mathrm{~S}$ rRNA gene amplicon sequencing were collected at 0 days post-starvation (0dpS), $1 \mathrm{dpS}, 3 \mathrm{dpS}, 7 \mathrm{dpS}, 21 \mathrm{dpS}, 1$ day post-re-feed $(\mathrm{dpR}), 3 \mathrm{dpR}, 7 \mathrm{dpR}$, and $21 \mathrm{dpR}$ (Fig. 1A) with six randomly selected fish at each time point per tank were euthanized by tricaine overdose $(0.83 \mathrm{mg} / \mathrm{ml}$ tricaine $)$. Fish were imaged on a dissecting scope to facilitate subsequent standard length (SL) and height at anterior of anal fin (HAA) measurements ${ }^{94}$. Intestinal tracts were then dissected from each fish and placed individually in lysis buffer $(20 \mathrm{mM}$ Tris- $\mathrm{HCl}(\mathrm{pH} 8.0), 2 \mathrm{mM}$ EDTA $(\mathrm{pH} 8.0), 1 \%$ Triton $\mathrm{X}-100$, flash-frozen in a dry-ice/ethanol bath, and stored at $-80^{\circ} \mathrm{C}$ until DNA extraction.

Samples for RNA-Seq were collected at 3dpS, 21dpS, and 3dpR At each time point, three randomly selected fish per tank were euthanized by tricaine overdose $(0.83 \mathrm{mg} / \mathrm{ml}$ tricaine $)$. Fish were imaged on a dissecting scope to facilitate subsequent standard length $(\mathrm{SL})^{94}$. Intestinal tracts were then dissected from each fish and placed individually in $2 \mathrm{~mL}$ cryovials filled with TRIzol reagent (Thermo Fisher, 15596026), flashfrozen in a dry ice-ethanol bath, and stored at $-80^{\circ} \mathrm{C}$ until RNA extraction.

\section{S rRNA gene sequencing}

Genomic DNA was extracted from individual zebrafish intestinal tracts using Qiagen DNeasy Blood and Tissue Kits (Qiagen, modified as previously described) ${ }^{42}$. Genomic DNA was subsequently used as template for PCR amplification of the v4 region of $16 \mathrm{~S}$ rRNA gene and 150 paired-end sequencing was performed on an Illumina HiSeq 2000 Sequencing System (see Table S4 for primers) at the University of Oregon 
FASTQ files were demultiplexed and split by sample ID using QIIME (v1.9.1). Within RStudio version 3.4.1, the files were then quality filtered, trimmed, denoised, merged, checked for chimeras, and assigned taxonomy using DADA2. Taxonomic assignments were made using the Silva v132 database. RNA extraction and sequencing

Frozen whole intestinal samples stored at $-80^{\circ} \mathrm{C}$ were homogenized using

\section{RNA-seq bioinformatics} Zirconium oxide beads (Biospec, 11079107) and a Vortex Genie2 (Scientific Industries, 1311-V) fitted with a Vortex Adapter (Scientific Industries, 13000-V1-24) in three 45second intervals. Samples were put on ice in-between homogenization to prevent overheating. Following homogenization, a phase separation was performed by adding $200 \mathrm{ul}$ of chloroform to each sample and mixing by vigorous inversion 15 times. Samples were then incubated at room temperature for 3 minutes and centrifuged at 12000 rcf for $15 \mathrm{~min}$ at $4^{\circ} \mathrm{C}$. 500ul of the aqueous upper phase from each sample was then transferred to a new Eppendorf tube, to which 500uL 70\% Ethanol in DEPC water was added and vortexed. Following phase separation, samples were DNase treated and total RNA was extracted via column purification using the PureLink DNase Set (Thermo Fisher, 12185010) and the PureLink RNA Mini kit (Thermo Fisher, 12183025) according to the manufacturer's instructions. Final sample quality and concentration were assessed via spectrophotometry and samples were stored at $-80^{\circ} \mathrm{C}$ until submission to the Duke Sequencing and Genomic Technologies Core. RNA-seq libraries were prepared and sequenced by Duke Sequencing and Genomic Technologies Core on an Illumina HiSeq 2500 .

All raw zebrafish RNA-seq data was processed on the Galaxy server ${ }^{95}$. Raw fastq files were trimmed using Trim Galore ${ }^{96}$. Trimmed fastq files were then mapped to the zebrafish genome (GRCz10) using STAR using default settings to generate BAM files, which were converted to counts using HTSeq. BAM files were converted to bigWig files using the wigToBigWig tool before visualization on the UCSC Genome Browser 97,98.

TPM expression values were obtained for transcripts via Salmon ${ }^{99}$. Pairwise differential gene expression tests were carried out with DESeq2 using counts files generated by HTSeq ${ }^{99,100}$. For comparisons between starved and fed fish, the default significance threshold of adjusted p-value 0.05 was used for each comparison. For comparisons across fed fish controls (See Fig. S1 and S2), the significance threshold was defined as the gene either having an absolute $\log _{2}$ fold change greater than 1.0 or a p-value less than 0.05 . 
Hierarchical clustering of $\log _{2}$ fold change values for genes was performed using Cluster 3.0, and heat maps were generated using Java Treeview ${ }^{101,102}$.

HOMER software (http://homer.ucsd.edu/homer/motif/) analysis was performed on significantly upregulated and downregulated genes at both $3 \mathrm{dpS}$ and $21 \mathrm{dpS}$ (3dpS starved/3dpS fed and $21 \mathrm{dpS}$ starved/21dpS fed, respectively), using regions within the gene body plus the flanking $10 \mathrm{~kb}$ upstream and downstream that we previously identified as accessible chromatin in the zebrafish intestine ${ }^{51}$ using the findMotifs.pl command. A motif score was obtained by taking the $-\log _{10}$ values of the $p$-values assigned by HOMER. A motif was then deemed 'enriched' amongst either upregulated or downregulated genes at each timepoint (3dpS or $21 \mathrm{dpS}$ ) based on whether it had a higher motif score among the upregulated or downregulated gene sets.

For comparisons with the larval zebrafish egg yolk feeding dataset, $\log _{2}$ fold changes in $21 \mathrm{dpS}$ fed fish relative to $21 \mathrm{dpS}$ starved were compared to $\log _{2}$ fold changes in larval zebrafish digestive tracts 4 hours after egg yolk feeding (i.e. "HF $4 \mathrm{~h}$ logFC") obtained from Supplementary Table 1 in ${ }^{59}$, the raw data for which is available at accession GSE87704. For comparisons with hnf4a mutant and microbially colonization datasets, data was obtained from Supplemental Table 2 in ${ }^{75}$, using $\log _{2}$ fold changes comparing digestive tracts from hnf4a homozygous mutant and wild-type $6 \mathrm{dpf}$ zebrafish larvae raised under conventionalized ex-germ-free consitions ("MutCV/WTCV") and from wild-type $6 \mathrm{dpf}$ zebrafish larvae reared under germ-free of ex-germ-free conventionalized conditions ("WTGF/WTCV"), respectively. Raw data from ${ }^{75}$ is available at accession GSE90462.

All zebrafish experiments were conducted in conformity with the Public Health Service Policy on Humane Care and Use of Laboratory Animals using protocols A165-13-06 and A115-16-05 approved by the Institutional Animal Care and Use Committee of Duke University. This study was completed in compliance with ARRIVE guidelines.

\section{Consent for publication}

Not applicable

\section{Availability of Data and Materials}

763

All quality filtering parameters for generating the sequence variants, ASV table and figures for the $16 \mathrm{~S}$ rRNA analysis can be found at: 
https://github.com/alexmccumber/fishguts. Data analysis used the $\mathrm{R}$ packages vegan and phyloseq. LEFsE was accessed through the Huttenhower Galaxy website: https://huttenhower.sph.harvard.edu/galaxyl. The raw 16S rRNA gene amplicon FASTQ files can be accessed from the European Nucleotide Archive under project access number PRJEB31503. Raw and processed RNA-Seq data is available on NCBI GEO at the accession GSE140821.

\section{Competing Interests}

The authors declare that they have no competing interests.

\section{Funding}

This work was supported by grants to B.J.M.B., K.G., and J.F.R from the NIH (R01GM095385) and the Gordon and Betty Moore Foundation; and to J.F.R from the NIH (R01-DK093399, R01-DK111857, and R01-DK081426). A.W.M. and C.A. were supported by the NSF Research Traineeship Program "Integrative Bioinformatics for Investigating and Engineering Microbiomes" or IBIEM (1545220). A.W.M. was also supported by the Center for Biomolecular and Tissue Engineering (NIH T32-GM008555).

\section{Authors Contributions}

J.R., K.G., B.B., S.W., and S.G. conceived and planned the study. S.W. and S.G. conducted the animal experiments and generated the data. A.M., J.J., C.L., and C.A. analyzed the data. A.M. and J.J. drafted the manuscript and generated the figures and tables. A.M., J.J., C.L., C.A., S.C., B.B., K.G., and J.R. edited the manuscript. All authors reviewed the manuscript. J.R. and C.L. supervised the project. J.R., K.G., and B.B. obtained the funding.

\section{Acknowledgements}

We are grateful to Dr. Joshua Granek who provided instruction as a part of IBIEM and that without we would have been unable to complete this work, and to Dr. Doug Turnbull

\section{References}

802 
1. Watts, M. J. \& Bohle, H. G. The space of vulnerability: the causal structure of hunger and

804 famine: Progress in Human Geography (2016) doi:10.1177/030913259301700103.

805 2. Fearon, K. et al. Definition and classification of cancer cachexia: an international

806 consensus. Lancet Oncol. 12, 489-495 (2011).

807 3. Nagy, K. Food requirements of wild animals: predictive equations for free-living mammals, 808 reptiles, and birds. (2018).

809 4. McCue, M. D. Starvation physiology: Reviewing the different strategies animals use to 810 survive a common challenge. Comparative Biochemistry and Physiology Part A: Molecular 811 \& Integrative Physiology 156, 1-18 (2010).

812 5. Storey, K. B. \& Storey, J. M. Metabolic Rate Depression and Biochemical Adaptation in 813 Anaerobiosis, Hibernation and Estivation. The Quarterly Review of Biology 65, 145-174 814 (1990).

815 6. Nagy, K. A., Girard, I. A. \& Brown, T. K. Energetics of free-ranging mammals, reptiles, and 816 birds. Annual Review of Nutrition; Palo Alto 19, 247-77 (1999).

817 7. Thomas, D. R. Loss of skeletal muscle mass in aging: examining the relationship of 818 starvation, sarcopenia and cachexia. Clinical nutrition 26, 389-399 (2007).

819 8. Love, A. H. Metabolic response to malnutrition: its relevance to enteral feeding. Gut 27, 9$82013(1986)$.

821 9. Moore, F. D. Energy and the maintenance of the body cell mass. Journal of Parenteral and 822 Enteral Nutrition 4, 228-260 (1980).

823 10. McFarlane, H. et al. Immunity, Transferrin, and Survival in Kwashiorkor. Br Med J 4, 268$824270(1970)$

825 11. Champakam, S., Srikantia, S. G. \& Gopalan, C. Kwashiorkor and Mental Development. Am 826 J Clin Nutr 21, 844-852 (1968).

827 12. Acheson, R. M. \& Macintyre, M. N. The Effects of Acute Infection and Acute Starvation on 828 Skeletal Development. Br J Exp Pathol 39, 37-45 (1958). 
13. Palesty, J. A. \& Dudrick, S. J. The Goldilocks Paradigm of Starvation and Refeeding. Nutrition in Clinical Practice 21, 147-154 (2006).

14. Cahill, G. F. Starvation in Man. New England Journal of Medicine 282, 668-675 (1970).

832 15. Felig, P., Owen, O. E., Wahren, J. \& Cahill, G. F. Amino acid metabolism during prolonged 833 starvation. J Clin Invest 48, 584-594 (1969).

834 16. Désert, C. et al. Transcriptome profiling of the feeding-to-fasting transition in chicken liver.

835 BMC Genomics 9, 611 (2008).

836 17. Drew, R. E. et al. Effect of starvation on transcriptomes of brain and liver in adult female 837 zebrafish (Danio rerio). Physiological Genomics 35, 283-295 (2008).

838 18. Hakvoort, T. B. M. et al. Interorgan coordination of the murine adaptive response to fasting.

839 J. Biol. Chem. jbc.M110.216986 (2011) doi:10.1074/jbc.M110.216986.

840 19. Crawford, P. A. et al. Regulation of myocardial ketone body metabolism by the gut

841 microbiota during nutrient deprivation. PNAS 106, 11276-11281 (2009).

842 20. David, L. A. et al. Diet rapidly and reproducibly alters the human gut microbiome. Nature $843 \quad 505,559-563(2014)$.

844 21. Hildebrandt, M. A. et al. High-fat diet determines the composition of the murine gut 845 microbiome independently of obesity. Gastroenterology 137, 1716-1724.e1-2 (2009).

846 22. Turnbaugh, P. J., Bäckhed, F., Fulton, L. \& Gordon, J. I. Diet-Induced Obesity Is Linked to 847 Marked but Reversible Alterations in the Mouse Distal Gut Microbiome. Cell Host \& Microbe $848 \quad 3,213-223(2008)$.

849 23. Basolo, A. et al. Effects of underfeeding and oral vancomycin on gut microbiome and 850 nutrient absorption in humans. Nature Medicine 1-10 (2020) doi:10.1038/s41591-020-0801851 z.

852 24. Backhed, F. et al. gut microbiota as an environmental factor that regulates fat storage.

853 Proceedings of the National Academy of Sciences of the United States of America (2004). 
25. Smith, M. I. et al. Gut Microbiomes of Malawian Twin Pairs Discordant for Kwashiorkor.

$855 \quad$ Science 339, 548-554 (2013).

856 26. Ridaura, V. K. et al. Gut microbiota from twins discordant for obesity modulate metabolism $857 \quad$ in mice. Science 341, 1241214 (2013).

858 27. Cowardin, C. A. et al. Mechanisms by which sialylated milk oligosaccharides impact bone 859 biology in a gnotobiotic mouse model of infant undernutrition. PNAS 116, 11988-11996 860 (2019).

861 28. Hibberd, M. C. et al. The effects of micronutrient deficiencies on bacterial species from the 862 human gut microbiota. Science Translational Medicine 9, eaal4069 (2017).

863 29. Gehrig, J. L. et al. Effects of microbiota-directed foods in gnotobiotic animals and $864 \quad$ undernourished children. Science 365, eaau4732 (2019).

865 30. Leulier, F. et al. Integrative Physiology: At the Crossroads of Nutrition, Microbiota, Animal 866 Physiology, and Human Health. Cell Metab. 25, 522-534 (2017).

867 31. Gas, N. \& Noailliac-Depeyre, J. Studies on intestinal epithelium involution during prolonged 868 fasting. Journal of Ultrastructure Research 56, 137-151 (1976).

869 32. Segner, H. \& Braunbeck, T. Hepatocellular adaptation to extreme nutritional conditions in 870 ide,Leuciscus idus melanotus L. (Cyprinidae). A morphofunctional analysis. Fish Physiol $871 \quad$ Biochem 5, 79-97 (1988).

872 33. Lenaerts, K. et al. Starvation Induces Phase-Specific Changes in the Proteome of Mouse 873 Small Intestine. J. Proteome Res. 5, 2113-2122 (2006).

874 34. Saotome, I., Curto, M. \& McClatchey, A. I. Ezrin Is Essential for Epithelial Organization and 875 Villus Morphogenesis in the Developing Intestine. Developmental Cell 6, 855-864 (2004).

876 35. Habold, C., Foltzer-Jourdainne, C., Le Maho, Y. \& Lignot, J.-H. Intestinal apoptotic changes 877 linked to metabolic status in fasted and refed rats. Pflugers Arch - Eur J Physiol 451, 749$878 \quad 759$ (2006). 
36. Goldsmith, M. I., lovine, M. K., O'Reilly-Pol, T. \& Johnson, S. L. A developmental transition in growth control during zebrafish caudal fin development. Dev. Biol. 296, 450-457 (2006).

37. Flynn, E. J., Trent, C. M. \& Rawls, J. F. Ontogeny and nutritional control of adipogenesis in zebrafish (Danio rerio). J. Lipid Res. 50, 1641-1652 (2009).

38. McMenamin, S. K., Minchin, J. E. N., Gordon, T. N., Rawls, J. F. \& Parichy, D. M. Dwarfism and increased adiposity in the gh1 mutant zebrafish vizzini. Endocrinology 154, 1476-1487 (2013).

39. Minchin, J. E. N. \& Rawls, J. F. A classification system for zebrafish adipose tissues. Dis Model Mech 10, 797-809 (2017).

40. Ng, A. N. et al. Formation of the digestive system in zebrafish: III. Intestinal epithelium morphogenesis. Developmental biology 286, 114-135 (2005).

41. Rawls, J. F., Samuel, B. S. \& Gordon, J. I. Gnotobiotic zebrafish reveal evolutionarily conserved responses to the gut microbiota. PNAS 101, 4596-4601 (2004).

42. Stephens, W. Z. et al. The composition of the zebrafish intestinal microbial community varies across development. The ISME Journal 10, 644-654 (2016).

43. Semova, I. et al. Microbiota Regulate Intestinal Absorption and Metabolism of Fatty Acids in the Zebrafish. Cell Host \& Microbe 12, 277-288 (2012).

44. Wong, S. et al. Ontogenetic Differences in Dietary Fat Influence Microbiota Assembly in the Zebrafish Gut. mBio 6, e00687-15 (2015).

45. Wallace, K. N. \& Pack, M. Unique and conserved aspects of gut development in zebrafish. Developmental biology 255, 12-29 (2003).

46. Cheesman, S. E., Neal, J. T., Mittge, E., Seredick, B. M. \& Guillemin, K. Epithelial cell proliferation in the developing zebrafish intestine is regulated by the Wnt pathway and microbial signaling via Myd88. Proc. Natl. Acad. Sci. U.S.A. 108 Suppl 1, 4570-4577 (2011). 
47. Bates, J. M. et al. Distinct signals from the microbiota promote different aspects of zebrafish 905 gut differentiation. Developmental Biology 297, 374-386 (2006).

48. Rolig, A. S., Parthasarathy, R., Burns, A. R., Bohannan, B. J. M. \& Guillemin, K. Individual Members of the Microbiota Disproportionately Modulate Host Innate Immune Responses. Cell Host \& Microbe 18, 613-620 (2015).

49. Murdoch, C. C. et al. Intestinal Serum amyloid A suppresses systemic neutrophil activation

50. Kanther, M. et al. Microbial Colonization Induces Dynamic Temporal and Spatial Patterns of

913 NF-kB Activation in the Zebrafish Digestive Tract. Gastroenterology 141, 197-207 (2011).

914 51. Lickwar, C. R. et al. Genomic dissection of conserved transcriptional regulation in intestinal 915 epithelial cells. PLOS Biology 15, e2002054 (2017).

916 52. Jia, J. et al. Microarray and metabolome analysis of hepatic response to fasting and 917 subsequent refeeding in zebrafish (Danio rerio). BMC Genomics 20, 919 (2019).

918 53. Minchin, J. E. N., Scahill, C. M., Staudt, N., Busch-Nentwich, E. M. \& Rawls, J. F. Deep 919 phenotyping in zebrafish reveals genetic and diet-induced adiposity changes that may 920 inform disease risk. J. Lipid Res. 59, 1536-1545 (2018).

921 54. Burns, A. R. et al. Contribution of neutral processes to the assembly of gut microbial 922 communities in the zebrafish over host development. ISME J 10, 655-664 (2016).

923 55. Rawls, J. F., Mahowald, M. A., Ley, R. E. \& Gordon, J. I. Reciprocal Gut Microbiota 924 Transplants from Zebrafish and Mice to Germ-free Recipients Reveal Host Habitat 925 Selection. Cell 127, 423-433 (2006).

926 56. Ar, B. et al. Interhost dispersal alters microbiome assembly and can overwhelm host innate 927 immunity in an experimental zebrafish model. Proceedings of the National Academy of 928 Sciences of the United States of America vol. 114

929 http://pubmed.ncbi.nlm.nih.gov/28973938/ (2017). 
57. Segata, N. et al. Metagenomic biomarker discovery and explanation. Genome Biology 12,

$931 \quad \mathrm{R} 60$ (2011).

932 58. Morais, S., Monroig, O., Zheng, X., Leaver, M. J. \& Tocher, D. R. Highly Unsaturated Fatty

933 Acid Synthesis in Atlantic Salmon: Characterization of ELOVL5- and ELOVL2-like

$934 \quad$ Elongases. Mar Biotechnol 11, 627-639 (2009).

935 59. Zeituni, E. M. et al. Endoplasmic reticulum lipid flux influences enterocyte nuclear

936 morphology and lipid-dependent transcriptional responses. J. Biol. Chem. jbc.M116.749358

937 (2016) doi:10.1074/jbc.M116.749358.

938 60. Wang, Y. et al. Tissue-specific, nutritional, and developmental regulation of rat fatty acid

939 elongases. J. Lipid Res. 46, 706-715 (2005).

940 61. Cruciani-Guglielmacci, C. et al. Molecular phenotyping of multiple mouse strains under

941 metabolic challenge uncovers a role for Elovl2 in glucose-induced insulin secretion.

942 Molecular Metabolism 6, 340-351 (2017).

943 62. Alimuddin, Kiron, V., Satoh, S., Takeuchi, T. \& Yoshizaki, G. Cloning and over-expression of

944 a masu salmon (Oncorhynchus masou) fatty acid elongase-like gene in zebrafish.

$945 \quad$ Aquaculture 282, 13-18 (2008).

946 63. Antalis, T. M., Shea-Donohue, T., Vogel, S. N., Sears, C. \& Fasano, A. Mechanisms of

947 Disease: protease functions in intestinal mucosal pathobiology. Nat Clin Pract Gastroenterol

$948 \quad$ Hepatol 4, 393-402 (2007).

949 64. Mukherjee, S. \& Hooper, L. V. Antimicrobial defense of the intestine. Immunity 42, 28-39

$950 \quad$ (2015).

951 65. Holzinger, A. et al. Mutations in the Proenteropeptidase Gene Are the Molecular Cause of

952 Congenital Enteropeptidase Deficiency. Am J Hum Genet 70, 20-25 (2002).

953 66. Thierer, J. H., Ekker, S. C. \& Farber, S. A. The LipoGlo reporter system for sensitive and 954 specific monitoring of atherogenic lipoproteins. Nature Communications 10, 3426 (2019). 
67. Salem, M., Silverstein, J., Rexroad, C. E. \& Yao, J. Effect of starvation on global gene expression and proteolysis in rainbow trout (Oncorhynchus mykiss). BMC Genomics 8, 328 (2007).

68. Lu, D.-L. et al. Fasting enhances cold resistance in fish through stimulating lipid catabolism and autophagy. The Journal of Physiology 597, 1585-1603 (2019).

69. Song, H., Yan, Y., Titus, T., He, X. \& Postlethwait, J. H. The role of stat1b in zebrafish hematopoiesis. Mechanisms of Development 128, 442-456 (2011).

70. Richmond, C. A. et al. JAK/STAT-1 Signaling Is Required for Reserve Intestinal Stem Cell Activation during Intestinal Regeneration Following Acute Inflammation. Stem Cell Reports 10, 17-26 (2017).

71. Dobson, J. T. et al. Carboxypeptidase A5 identifies a novel mast cell lineage in the zebrafish providing new insight into mast cell fate determination. Blood 112, 2969-2972 (2008).

72. Hindle, A. G., Grabek, K. R., Epperson, L. E., Karimpour-Fard, A. \& Martin, S. L. Metabolic changes associated with the long winter fast dominate the liver proteome in 13-lined ground squirrels. Physiol Genomics 46, 348-361 (2014).

73. Kamata, S. et al. 2D DIGE proteomic analysis reveals fasting-induced protein remodeling through organ-specific transcription factor(s) in mice. FEBS Open Bio 8, 1524-1543 (2018).

74. Camp, J. G. et al. Microbiota modulate transcription in the intestinal epithelium without remodeling the accessible chromatin landscape. Genome Res. gr.165845.113 (2014) doi:10.1101/gr.165845.113.

75. Davison, J. M. et al. Microbiota regulate intestinal epithelial gene expression by suppressing the transcription factor Hepatocyte nuclear factor 4 alpha. Genome Res. 27, 1195-1206 (2017).

76. Larsson, E. et al. Analysis of gut microbial regulation of host gene expression along the length of the gut and regulation of gut microbial ecology through MyD88. Gut 61, 11241131 (2012). 
77. Heinz, S. et al. Simple Combinations of Lineage-Determining Transcription Factors Prime cis-Regulatory Elements Required for Macrophage and B Cell Identities. Molecular Cell 38, 576-589 (2010).

78. Xu, H. et al. Transcriptional inhibition of intestinal NHE8 expression by glucocorticoids involves Pax5. American Journal of Physiology-Gastrointestinal and Liver Physiology 299, G921-G927 (2010).

79. Kaestner, K. H. Making the liver what it is: The many targets of the transcriptional regulator HNF4a. Hepatology 51, 376-377 (2010).

80. Roman, A. K. S., Aronson, B. E., Krasinski, S. D., Shivdasani, R. A. \& Verzi, M. P. Transcription Factors GATA4 and HNF4A Control Distinct Aspects of Intestinal Homeostasis in Conjunction with Transcription Factor CDX2. J. Biol. Chem. 290, 1850-1860 (2015).

81. Palanker, L., Tennessen, J. M., Lam, G. \& Thummel, C. S. Drosophila HNF4 Regulates Lipid Mobilization and $\beta$-Oxidation. Cell Metabolism 9, 228-239 (2009).

82. Frochot, V. et al. The transcription factor HNF-4a: a key factor of the intestinal uptake of fatty acids in mouse. American Journal of Physiology-Gastrointestinal and Liver Physiology 302, G1253-G1263 (2012).

83. Barry, W. E. \& Thummel, C. S. The Drosophila HNF4 nuclear receptor promotes glucosestimulated insulin secretion and mitochondrial function in adults. Elife 5, (2016).

84. Xia, J. H. et al. The intestinal microbiome of fish under starvation. BMC Genomics 15, 266 (2014).

85. Rescan, P.-Y. et al. Dynamic gene expression in fish muscle during recovery growth induced by a fasting-refeeding schedule. BMC Genomics 8, 438 (2007).

1004

86. Connor, E. E. et al. Enhanced mitochondrial complex gene function and reduced liver size may mediate improved feed efficiency of beef cattle during compensatory growth. Funct 1005 Integr Genomics 10, 39-51 (2010). 
1006

1007

1008

1009

1010

1011

1012

1013

1014

1015

1016

1017

1018

1019

1020

1021

1022

1023

1024

1025

1026

1027

1028

1029

1030

87. Rescan, P.-Y., Le Cam, A., Rallière, C. \& Montfort, J. Global gene expression in muscle from fasted/refed trout reveals up-regulation of genes promoting myofibre hypertrophy but not myofibre production. BMC Genomics 18, 447 (2017).

88. Carey, H. V., Walters, W. A. \& Knight, R. Seasonal restructuring of the ground squirrel gut microbiota over the annual hibernation cycle. Am. J. Physiol. Regul. Integr. Comp. Physiol. 304, R33-42 (2013).

89. Roeselers, G. et al. Evidence for a core gut microbiota in the zebrafish. The ISME Journal 5, 1595-1608 (2011).

90. Schroeder, B. O. Fight them or feed them: how the intestinal mucus layer manages the gut microbiota. Gastroenterol Rep (Oxf) 7, 3-12 (2019).

91. Smirnov, A., Sklan, D. \& Uni, Z. Mucin dynamics in the chick small intestine are altered by starvation. J. Nutr. 134, 736-742 (2004).

92. Szabady, R. L., Yanta, J. H., Halladin, D. K., Schofield, M. J. \& Welch, R. A. TagA is a secreted protease of Vibrio cholerae that specifically cleaves mucin glycoproteins. Microbiology, 157, 516-525 (2011).

93. Bhowmick, R. et al. Intestinal Adherence of Vibrio cholerae Involves a Coordinated Interaction between Colonization Factor GbpA and Mucin. Infection and Immunity 76, 49684977 (2008).

94. Parichy, D. M., Elizondo, M. R., Mills, M. G., Gordon, T. N. \& Engeszer, R. E. Normal table of postembryonic zebrafish development: Staging by externally visible anatomy of the living fish. Developmental Dynamics 238, 2975-3015 (2009).

95. Afgan, E. et al. The Galaxy platform for accessible, reproducible and collaborative biomedical analyses: 2018 update. Nucleic Acids Res 46, W537-W544 (2018).

96. Krueger, F. Trim galore. A wrapper tool around Cutadapt and FastQC to consistently apply quality and adapter trimming to FastQ files (2015). 
1031 97. Casper, J. et al. The UCSC Genome Browser database: 2018 update. Nucleic Acids Res 1032 46, D762-D769 (2018).

1033 98. Dobin, A. et al. STAR: ultrafast universal RNA-seq aligner. Bioinformatics 29, 15-21 (2013).

1034 99. Patro, R., Duggal, G., Love, M. I., Irizarry, R. A. \& Kingsford, C. Salmon provides fast and 1035 bias-aware quantification of transcript expression. Nature Methods 14, 417-419 (2017).

1036 100. Love, M. I., Huber, W. \& Anders, S. Moderated estimation of fold change and dispersion 1037 for RNA-seq data with DESeq2. Genome Biology 15, 550 (2014).

1038 101. Saldanha, A. J. Java Treeview-extensible visualization of microarray data.

1039 Bioinformatics 20, 3246-3248 (2004).

1040 102. de Hoon, M. J. L., Imoto, S., Nolan, J. \& Miyano, S. Open source clustering software.

1041 Bioinformatics 20, 1453-1454 (2004).

1042

1043 


\section{Figures}

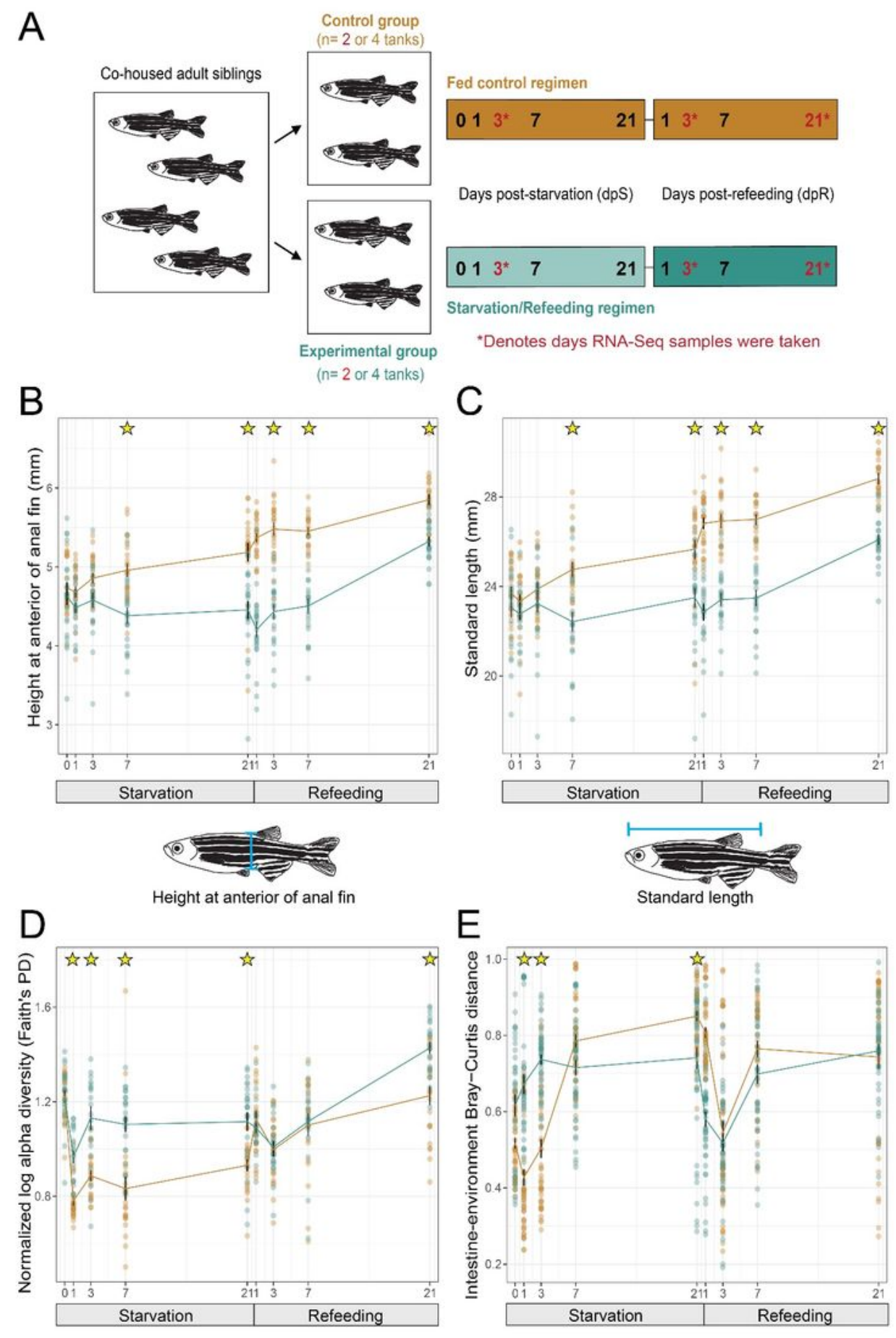

\section{Figure 1}

Starvation and refeeding affect zebrafish somatic growth as well as intestinal and environmental microbiome diversity. (A) Study design graphical abstract. Cohoused adult siblings were divided into either control (fed) or experimental (starved) tanks. Samples were then taken from each tank on days 0, 1 , 
3, 7 and 21 post-starvation (dpS) as well as 1, 3, 7, and 21 days post-refeeding (dpR) for 16S rRNA gene sequencing. RNA-seq samples were taken at $3 \mathrm{dpS}, 21 \mathrm{dpS}$, and $3 \mathrm{dpR}$. (B) Fed and starved zebrafish height at anterior of anal fin (HAA) in mm at corresponding timepoints. (C) Standard length in $\mathrm{mm}$ of starved and fed zebrafish. (D) Faith's PD alpha diversity for fed and control zebrafish. Values are log transformed and normalized by the scores at day 0. (E) Boxplots of the Bray-Curtis distance between the gut and associated environment sample. Stars in panels B-E denote significant difference $(p<0.05$ by Tukey HSD test).

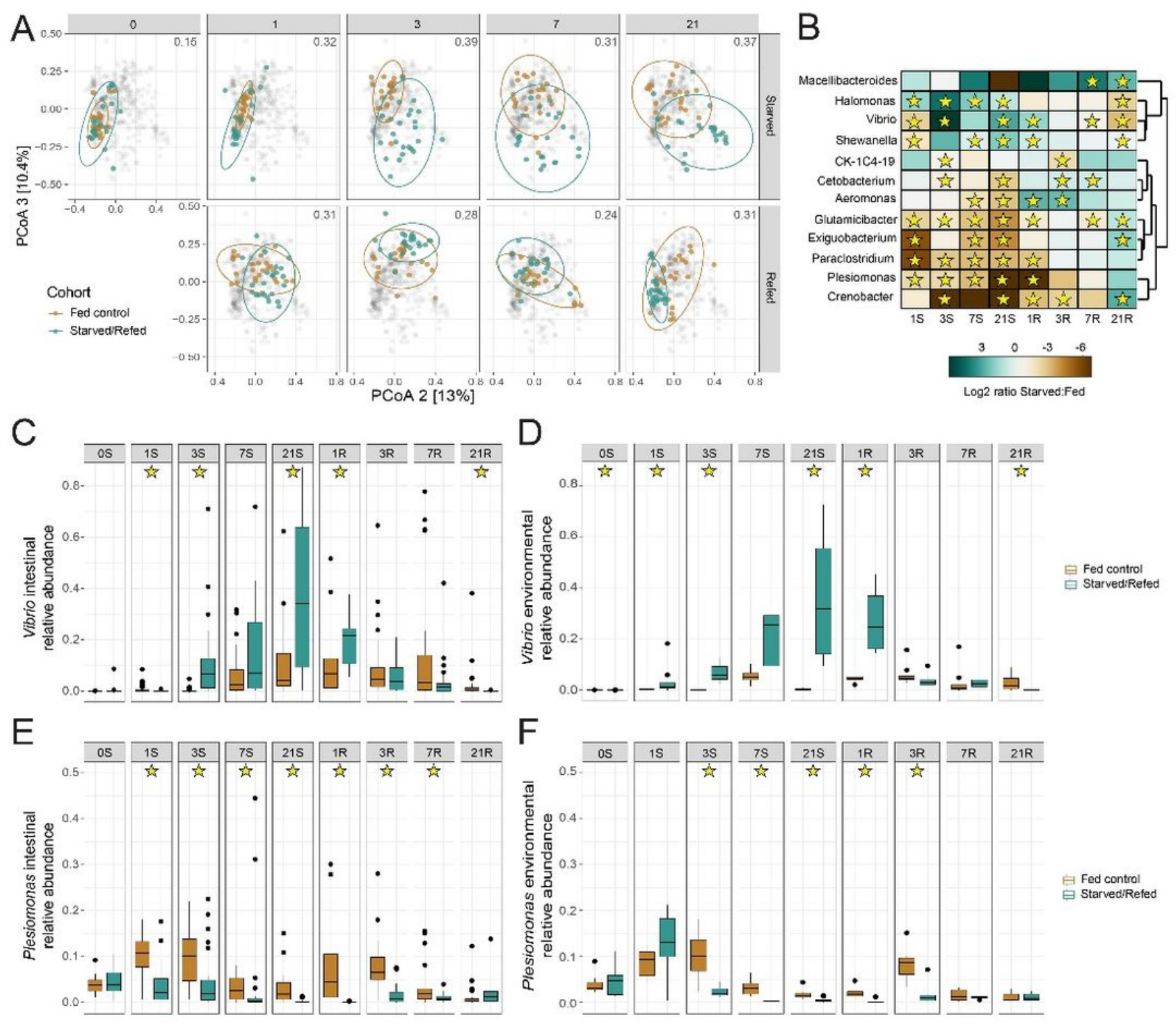

Figure 2

Starvation and refeeding dynamically alters membership of the adult zebrafish intestinal microbiome. (A) Principal coordinates analysis of Bray-Curtis diversity for fed and starved zebrafish. The distance between centroids of the two cohorts at the corresponding timepoint is shown in the top right of each plot. (B) Heatmap of log2 ratio of the relative abundance of bacterial genera between starved and fed 
controls. Stars denote day identified as significant by LEfSe. (C) Relative abundance of Vibrio in starved and control zebrafish intestines by day. (D) Relative abundance of Vibrio in starved and control environmental tank water samples by day. (E) Relative abundance of Plesiomonas in starved and control zebrafish intestines by day. (F) Relative abundance of Plesiomonas in starved and control environmental tank water samples by day. Stars in panels C-F denote significance $(p<0.05)$ by pairwise Wilcoxon test with $\mathrm{BH}$ correction.

A

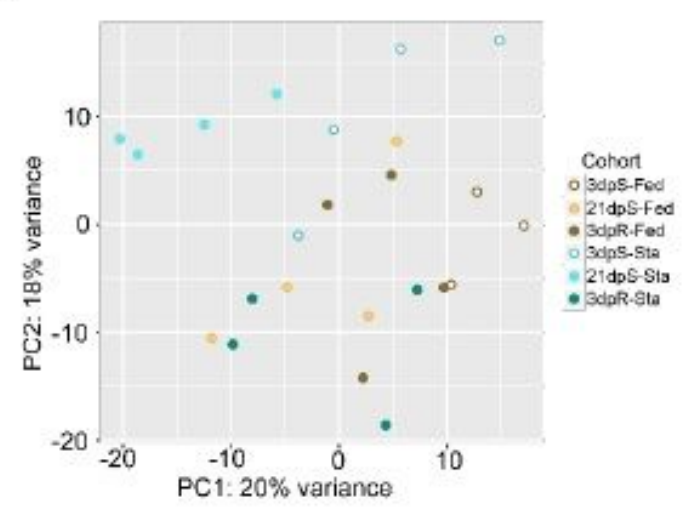

B

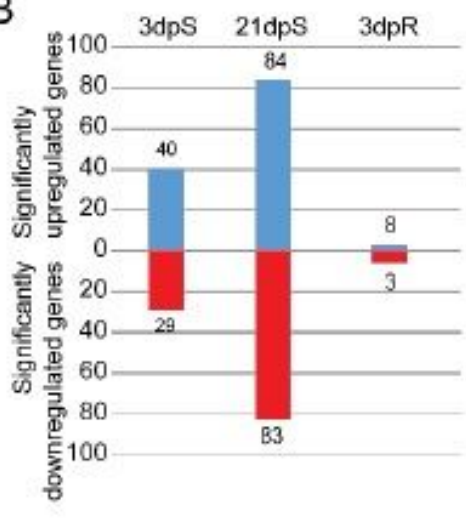

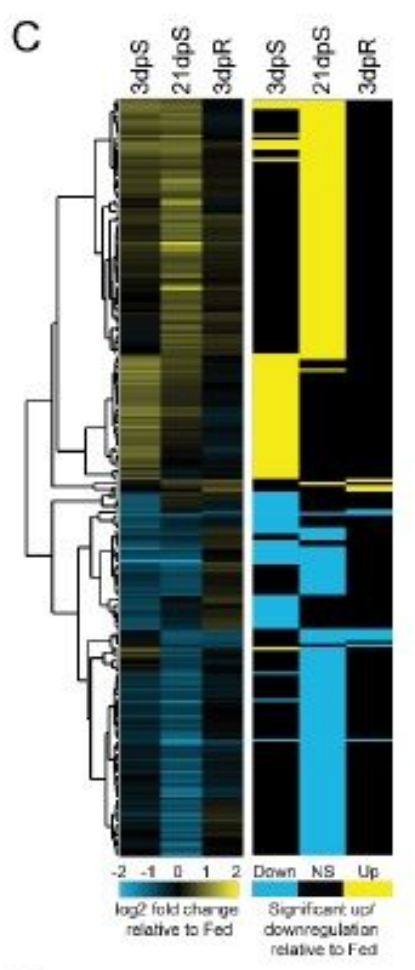

$E$

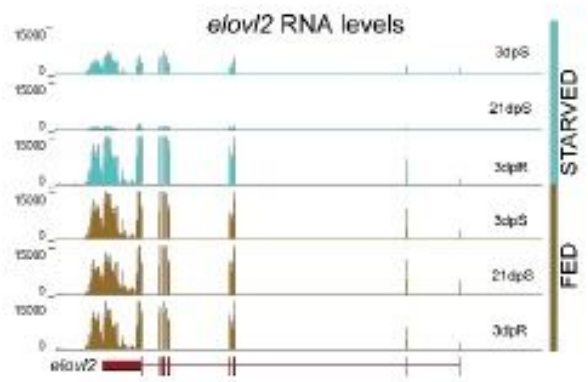

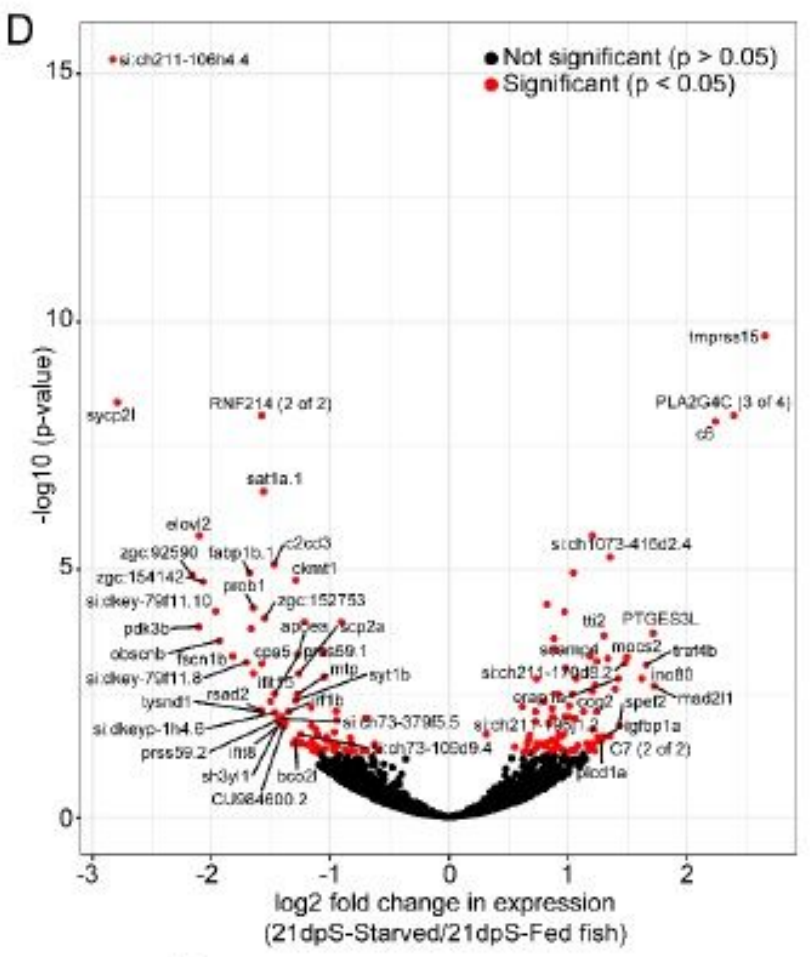

F

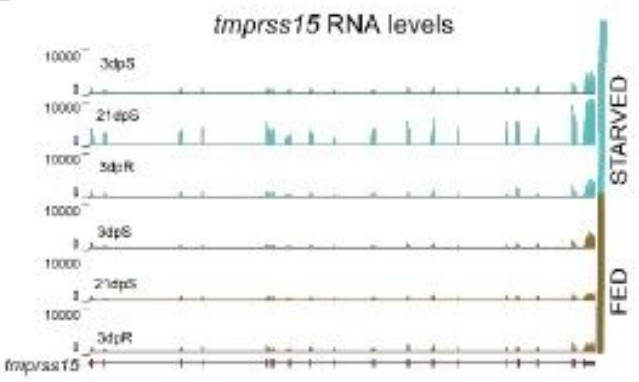


Starved zebrafish differentially regulate intestinal gene expression when compared to fed zebrafish (A) PCoA analysis of RNA-Seq libraries in starved/refed and fed control zebrafish intestines at 3dpS, $21 \mathrm{dpS}$, and 3dpR. (B) Quantification of the number of significantly upregulated and downregulated genes in starved/refed zebrafish intestines at each timepoint. Note that these numbers reflect totals after removing genes that were also significantly changed in our fed control comparisons. (C) Hierarchical clustering of log2 fold changes in gene expression in starved zebrafish intestines, along with flattened values that show significant changes in gene expression. (D) Log2 fold changes in gene expression in starved zebrafish intestines at $21 \mathrm{dpS}$ when compared to $21 \mathrm{dpS}$ fed fish plotted according to their -log10 adjusted p-values.(E) UCSC tracks of representative replicates show that elovl 2 mRNA, encoding a fatty acid elongase, is downregulated in starved zebrafish intestines and returns to levels comparable to the fed group upon re-feeding. (F) UCSC tracks of representative replicates show that tmprss 15 mRNA, encoding an enteropeptidase, is upregulated in starved zebrafish intestines and returns to levels comparable to the fed group upon re-feeding. 

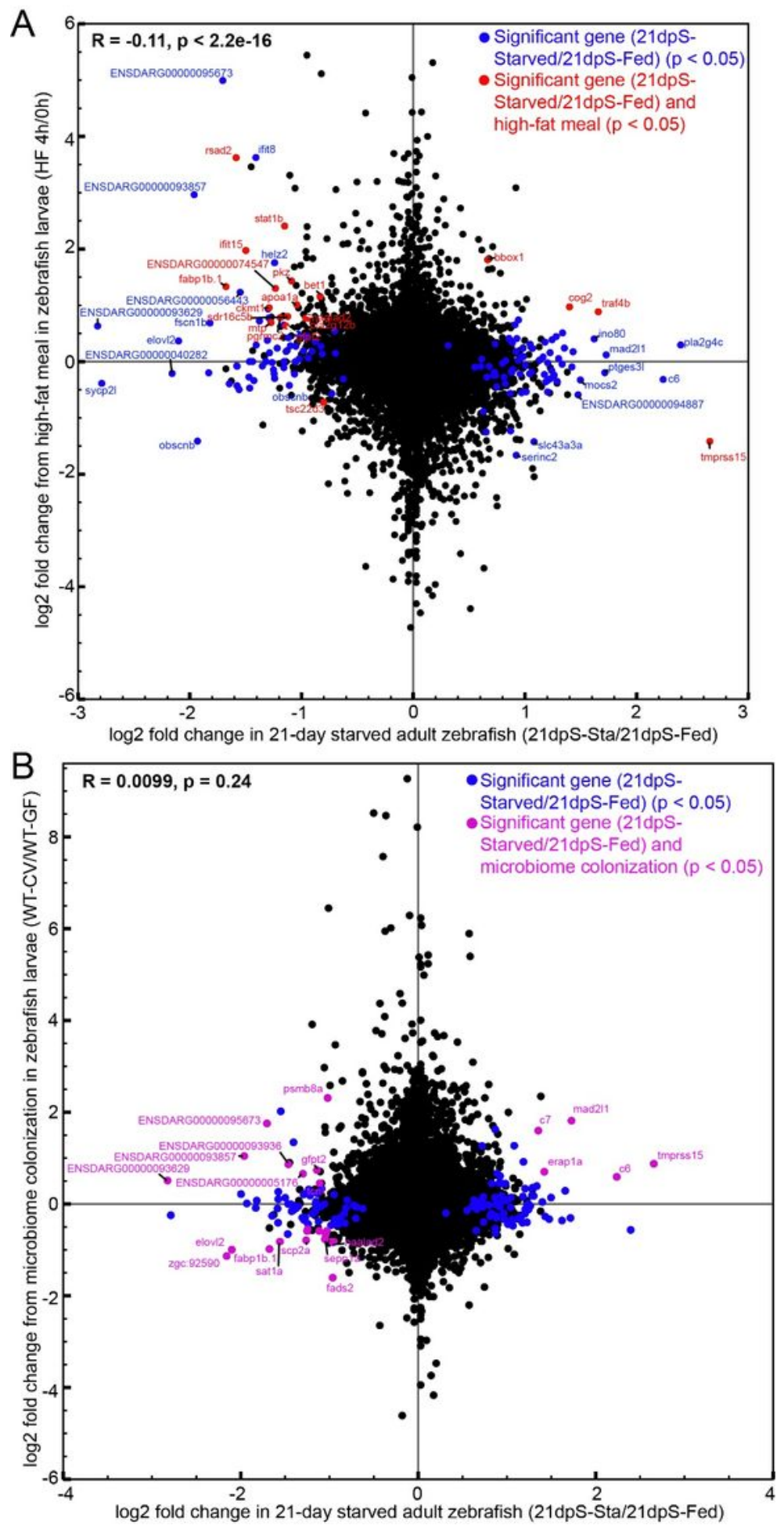

\section{Figure 4}

Some genes responsive to starvation in the intestine are 462 also responsive to high fat feeding and microbial colonization (A) Log2 fold changes for genes from $21 \mathrm{dpS}$ (X-axis) plotted according to their log2 fold changes in egg yolk-fed larval zebrafish compared to unfed controls ( $Y$-axis), described in Zeituni et al 59. Significantly differential genes only in starved zebrafish are plotted in blue, whereas genes significant in both datasets are plotted in red. Pearson's correlation revealed a significant 
correlation between the two datasets $(p<0.05)$. (B) Log2 fold changes for genes from $21 \mathrm{dpS}$ (X-axis) plotted according to their log2 fold changes in zebrafish larvae colonized with a microbiome compared to germ-free controls ( $\mathrm{Y}$-axis), described in Davison et al 75. Genes with significant log2 fold changes only in starved zebrafish are plotted in blue, whereas genes significant in both datasets are plotted in magenta. Pearson's correlation did not reveal a significant correlation between the two datasets $(p>0.05)$.

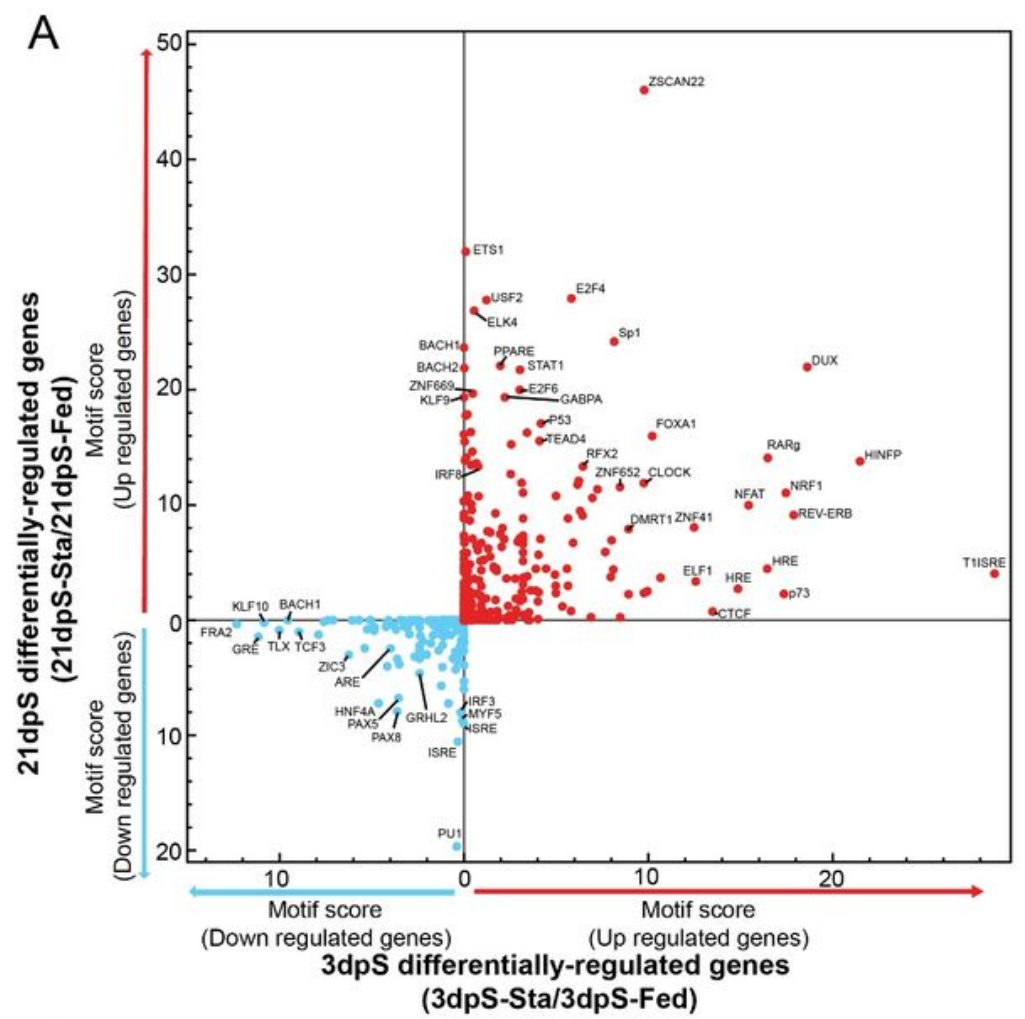

B

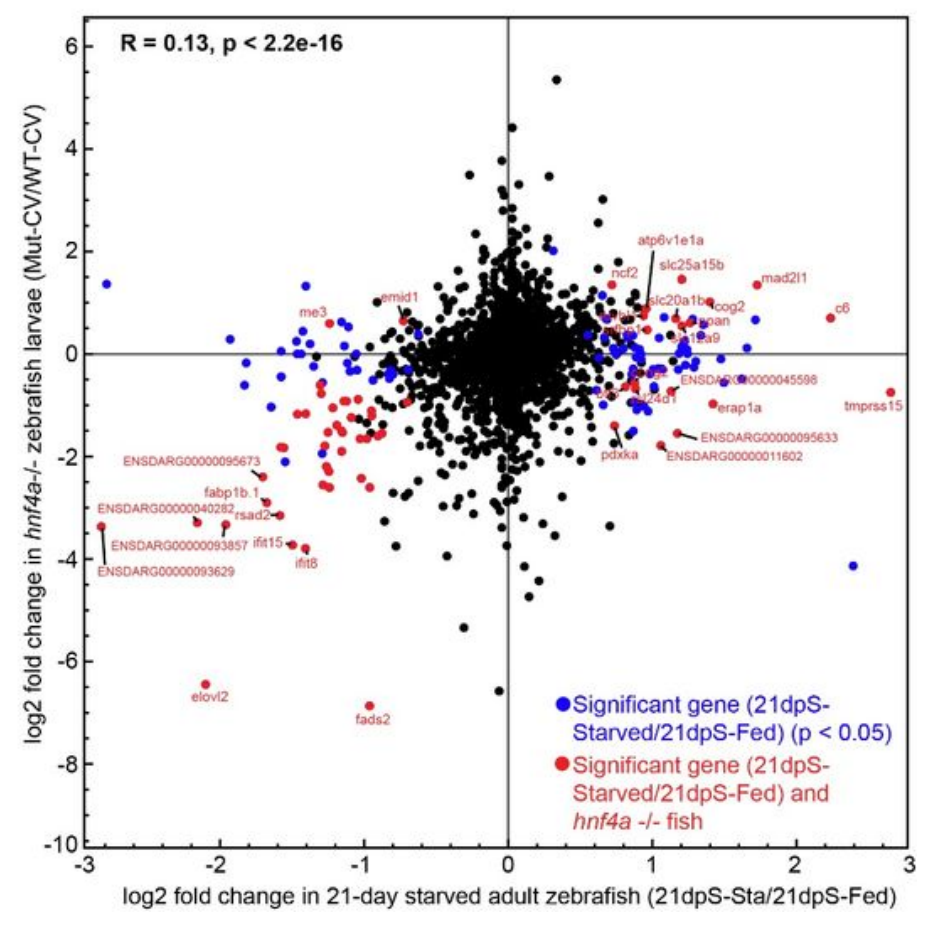

Figure 5 
The transcription factor hnf4a may regulate a subset of genes involved in starvation (A) Scatterplot for motif enrichment scores for genes at 3dps (X-axis) and motif enrichment scores for genes at $21 \mathrm{dpS}(\mathrm{Y}$ axis), according to HOMER analysis of transcription factor binding sites within 10KB upstream or downstream of the genes' transcription start sites at each time point, based on whether these sites were located within accessible chromatic regions. HNF4A is among the transcription factors whose binding sites are enriched at genes downregulated at both $3 \mathrm{dpS}$ and $21 \mathrm{dpS}$. (B) Log2 fold changes for genes from $21 \mathrm{dpS}$ (X-axis) plotted according to their log2 fold changes in digestive tracts dissected from hnf4a mutant zebrafish larvae compared to wild-type controls (Mut-CV/WT-CV) (Y-axis), described in Davison et al 75. Genes with genes with significant differential gene expression (21dpsSta/Fed) changes only in starved zebrafish are plotted in blue, whereas genes significant in both datasets are plotted in red. Pearson's correlation revealed a significant correlation between the two datasets $(p<0.05)$.

\section{Supplementary Files}

This is a list of supplementary files associated with this preprint. Click to download.

- FiguresS1andS2.pdf

- TableS1.xIsx

- TableS2.xIsx

- TableS3.xIsx

- TableS4.xIsx 